REVIEW ARTICLE

\title{
A careful reassessment of anthracycline use in curable breast
}

\section{cancer}

Sara Alsterlind Hurvitz $\mathbb{B}^{1 凶}$, Nicholas P. McAndrew $\mathbb{D}^{1}$, Aditya Bardia ${ }^{2}$, Michael F. Press ${ }^{3}$, Mark Pegram $\mathbb{C}^{4}$, John P. Crown ${ }^{5}$, Peter A. Fasching $\mathbb{1 0}^{6}$, Bent Ejlertsen $\mathbb{1}^{7}$, Eric H. Yang $\mathbb{D}^{1}$, John A. Glaspy $\mathbb{1 0}^{1}$ and Dennis J. Slamon ${ }^{1}$

It has been over three decades since anthracyclines took their place as the standard chemotherapy backbone for breast cancer in the curative setting. Though the efficacy of anthracycline chemotherapy is not debatable, potentially life-threatening and long-term risks accompany this class of agents, leading some to question their widespread use, especially when newer agents with improved therapeutic indices have become available. Critically assessing when to incorporate an anthracycline is made more relevant in an era where molecular classification is enabling not only the development of biologically targeted therapeutics but also is improving the ability to better select those who would benefit from cytotoxic agents. This comprehensive analysis will present the problem of overtreatment in early-stage breast cancer, review evidence supporting the use of anthracyclines in the pre-taxane era, analyze comparative trials evaluating taxanes with or without anthracyclines in biologically unselected and selected patient populations, and explore published work aimed at defining anthracycline-sensitive tumor types.

npj Breast Cancer (2021)7:134; https://doi.org/10.1038/s41523-021-00342-5

\section{INTRODUCTION: THE UNAVOIDABLE PROBLEM OF OVERTREATMENT IN EARLY-STAGE DISEASE}

\section{"As to diseases, make a habit of two things-to help, or at least to do no harm" Hippocrates}

Since the first publications of cytotoxic chemotherapy for earlystage breast cancer well over five decades ago ${ }^{1,2}$, numerous chemotherapeutic agents and regimens have been developed and tested with varying success for improvement in long-term outcomes. Compelling data from multiple studies including metaanalyses have concluded that adjuvant systemic chemotherapy reduces the risk of metastatic recurrence and improves overall survival $(\mathrm{OS})^{3}$. However, it is clear that not all patients require chemotherapy to become and remain cancer free. Although risk stratification of patients has become better using more sophisticated assessment of clinical risk, including subtyping of breast cancer and more recent use of genomic assays, there is still no completely accurate way to distinguish those patients who are rendered truly "disease free" by local measures vs those who have microscopic metastases and could benefit from cytotoxic therapy. This challenge has led to the overtreatment of many women.

Over $90 \%$ of breast cancer is localized to the breast and regional lymph nodes (LNs) at diagnosis ${ }^{4}$. Random assignment trials utilizing surgery alone as a control arm demonstrated that $75 \%$ of patients with negative axillary nodes are recurrence free at 10 years without systemic therapy ${ }^{5}$. A large meta-analysis of trials comparing adjuvant chemotherapy to no chemotherapy including all breast cancer subtypes, indicated that over $50 \%$ of patients will be free of cancer recurrence at 10 years in the absence of chemotherapy ${ }^{3}$. Although hormone receptor-negative (HR-) breast cancer is considered to be more aggressive, up to two- thirds of patients with HR- cancer confined to the breast will be cancer free at 16 years without any systemic therapy ${ }^{6}$. Moreover, while the benefits of chemotherapy in patients with regional LN involvement has been consistently demonstrated, roughly onequarter of these patients are disease free at 10 years with surgery alone $^{5,7}$. In order to abide by the primary guiding principle of medical ethics-avoiding harm to the patient-clinicians are charged with the daunting task of not only identifying patients most likely to benefit from chemotherapy but also utilizing the systemic regimen that has the highest short-term therapeutic index possible combined with the minimum risk of long-term, significant, treatment-related toxicities. This goal is made all the more challenging to achieve as physicians and patients, alike, want to avoid a recurrence and thus will often err on the side of overtreatment.

Advances in molecular characterization and biological subtyping have provided the opportunity to develop more targeted and effective, less toxic interventions for several breast cancer subgroups. In the early days of adjuvant systemic therapy, clinical trials exercised no molecular selection. Thus, patients with HRnegative breast cancer were included in endocrine therapy studies $^{8-12}$. These trials were generally positive, owing to the fact that the majority of breast cancers express HR, but we now know that patients with HR-negative disease do not benefit significantly from the addition of endocrine therapy ${ }^{13}$. The molecular classification of breast cancer is also facilitating new interpretations of results generated from randomized studies of systemic chemotherapy in which all subtypes of breast cancer were included without molecular selection. One such observation, based on the analysis of multiple studies comparing anthracycline to non-anthracycline regimens, is that the incremental benefit

\footnotetext{
${ }^{1}$ Department of Medicine, David Geffen School of Medicine, University of California, Los Angeles, Jonsson Comprehensive Cancer Center, Los Angeles, CA, USA. ${ }^{2}$ Massachusetts General Hospital, Harvard Medical School, Boston, MA, USA. ${ }^{3}$ University of Southern California, Los Angeles, CA, USA. ${ }^{4}$ Stanford Comprehensive Cancer Institute, Palo Alto, CA, USA. ${ }^{5}$ Department of Medical Oncology, St. Vincent's University Hospital, Dublin, Ireland. ${ }^{6}$ Department of Gynecology and Obstetrics, University Hospital Erlangen, FriedrichAlexander University Erlangen-Nuremberg, Comprehensive Cancer Center Erlangen-EMN, Erlangen, Germany. ${ }^{7}$ Department of Oncology, Rigshospitalet, Copenhagen University Hospital, Copenhagen, Denmark. ${ }^{凶}$ email: shurvitz@mednet.ucla.edu
} 
imparted by anthracyclines is likely restricted to a small subset of breast cancer.

In this review, we conduct a comprehensive analysis of the published literature relating to anthracyclines in early-stage breast cancer in order to define an evidence-based approach to the use of these agents in the curative setting.

\section{THE BENEFIT OF ANTHRACYCLINES IN UNSELECTED PATIENTS IN THE ERA PRIOR TO MOLECULAR SUBCLASSIFICATION}

Over a half-century has passed since Fisher and colleagues first evaluated perioperative cytotoxic chemotherapy for breast cancer in an attempt to reduce the risk of metastatic recurrence after radical mastectomy ${ }^{1}$. While the study was considered negative, multiple subsequent trials were conducted to evaluate systemic agents and regimens in the adjuvant setting. In several of these, significant improvements in relapse-free survival (RFS) and OS were observed ${ }^{14}$. Beginning in the 1970s, Bonadonna and colleagues evaluated the CMF regimen (cyclophosphamide, methotrexate, $5^{\prime}$-fluorouracil) in a series of clinical trials ${ }^{15}$. With over two decades of median follow up, these studies definitively demonstrated significant increases in RFS and OS with CMF compared to surgery alone.

Shortly after the FDA's approval of doxorubicin in $1974^{16}$, Stephen Jones and colleagues published the results of their trial evaluating the novel combination of doxorubicin and cyclophosphamide $(A C)$ for metastatic breast cancer $(M B C)^{17}$, based on Jones' preclinical demonstration of synergy between these two agents over a wide range of doses and schedules in vitro and in vivo ${ }^{18}$. Impressive responses were noted, with $80 \%$ experiencing an objective response, including six complete responses (CR). While this regimen was clearly active, two patients experienced congestive heart failure (CHF) after receiving a cumulative dose of $550 \mathrm{mg} / \mathrm{m}^{2}$ of doxorubicin, and one patient developed ST-T wave changes after a cumulative dose of $265 \mathrm{mg} / \mathrm{m}^{2}$, leading the authors to question whether an alternative schedule or dosing regimen would improve safety.

Subsequent to the Jones ${ }^{17}$ publication, a number of clinical trials were conducted to compare anthracycline-based regimens to non-anthracycline regimens and suggested anthracyclines may improve outcomes in the metastatic setting ${ }^{19-21}$. Based on these results, in the early 1980s a prospective randomized study in locally advanced, nonmetastatic breast cancer was initiated to evaluate postoperative CMF plus vincristine and prednisone (CMFVP) for 12 months vs CAF (cumulative dose of $300 \mathrm{mg} / \mathrm{m}^{2}$ doxorubicin) for 6 months followed by CMFVP for 6 months ${ }^{22}$. In this small study $(N=41)$, DFS trended toward improvement in anthracycline-treated patients $(p=0.05)$. Since then, at least 20 randomized studies ${ }^{9,23-34}$ (Supplementary Table 1A, B) have compared anthracycline to non-anthracycline-based adjuvant therapies. Of these, 13 studies which collectively enrolled 12,075 participants showed no benefit with the use of an anthracycline (Supplementary Table $1 \mathrm{~A})^{9,23-34}$, three ${ }^{23,35,36}(N=1883)$ showed a benefit in DFS but not in OS and four ${ }^{37-40}(N=4859)$ showed a benefit in both DFS and OS with the use of an anthracycline (Supplementary Table 1B). These studies differed in their inclusion of $\mathrm{LN}+$ or $\mathrm{LN}-$ disease, the inclusion of $\mathrm{HR}+$ or $\mathrm{HR}-$ breast cancer, allowance of endocrine therapy, and inclusion of premenopausal and postmenopausal patients. Importantly, these studies were conducted prior to the routine use of HER2 testing, thus the patient population was mixed and no one with HER2positive $(\mathrm{HER} 2+)$ cancer received trastuzumab. As will be discussed later, this latter point is critical because HER2- amplified cancers that have co-amplification of the topoisomerase 2 gene locus-TOP2A, may be uniquely sensitive to anthracyclines.

In 2005, the EBCTCG reported a meta-analysis from randomized trials begun prior to 1996 of systemic therapy (chemotherapy, endocrine therapy, or chemoendocrine therapy) for early breast cancer ${ }^{41}$. Over 14,000 women were included in studies comparing anthracycline-containing regimens to CMF-based regimens. This meta-analysis demonstrated a statistically significant $0.8 \%$ per year difference in recurrence rate and a $3.1 \%$ absolute improvement in 10-year mortality associated with anthracycline-based therapy. These data indicated that anthracyclines were associated with an $11 \%$ improvement in relative risk of recurrence and a $16 \%$ improvement in relative risk of death compared to CMF-based therapy. In 2012, this meta-analysis was updated ${ }^{3}$ and showed that compared to standard CMF, four cycles of standard AC chemotherapy $(N=5122)$ was not associated with a significant difference in 10 -year risk of recurrence $(42.1 \%$ CMF vs $41.0 \% \mathrm{AC}$, $p=0.76)$ nor improvement in breast cancer mortality $(32.5 \%$ CMF vs $31.6 \% \mathrm{AC}, p=0.67)(N=5000)$. A small but significant benefit was seen with the use of higher cumulative anthracycline dosage compared to CMF $(N=9527)$ in terms of recurrence (CMF $33.8 \%$ vs $31.2 \%$ anthracycline, $p=0.003$ ) and breast cancer mortality ( $24.1 \%$ CMF vs $20 \%$ anthracycline, $p=0.00001$ ). Again, HER2 status was neither available nor considered at the time of reporting these data.

In summary, some studies demonstrated that adjuvant anthracyclines were superior, but inconsistently, and then only slightly better than CMF. A meta-analysis was required to confirm this benefit. The ability to distinguish those patients who were receiving a large benefit from anthracyclines from those who received no benefit was obscured by the fact that none of the prospective studies stratified by HER2 status, several did not include endocrine therapy for $\mathrm{HR}+$ disease and trastuzumabbased therapy was not available for patients with HER2+ disease at the time these studies were conducted.

\section{THE ADVENT OF TAXANES}

A collaborative effort in the 1960 s-70s by the National Cancer Institute with the US Department of Agriculture in which thousands of plants were screened for anticancer activity led to the discovery of the first taxane, paclitaxel, from the bark of the Pacific Yew, paving the way to the development of docetaxel, a semi-synthetic analog from the renewable and more readily available leaves of the European yew tree ${ }^{42-44}$. Both drugs were shown to be highly active in MBC. Chan et al. ${ }^{45}$ demonstrated that docetaxel produced a significantly higher objective response rate than doxorubicin in a randomized trial in metastatic disease, the only agent shown to be superior to anthracycline in this setting. In this study, doxorubicin was associated with a $5 \%$ incidence of cardiac death.

This prominent activity of the taxanes in metastatic disease prompted early and extensive study of these drugs in the adjuvant setting. In contrast to the findings from many individual randomized studies of anthracycline-based therapy that showed no advantage in DFS or OS compared to CMF, the majority of individually randomized trials evaluating the use of taxanes in the curative setting were positive ${ }^{46-51}$, though there were a few exceptions ${ }^{52,53}$. Unlike the situation with anthracyclines, a metaanalysis was not needed to demonstrate the benefit of taxanes in the adjuvant treatment of breast cancer. Nevertheless, several meta-analyses were conducted, confirming that the addition of a taxane to anthracycline-based therapy significantly reduced the risk of recurrence and death ${ }^{3,54,55}$. Unfortunately, the lack of a non-anthracycline, taxane-based control arm in these early studies prevented the critical assessment of whether anthracyclines added any benefit to a taxane-based regimen.

Had taxanes been developed before anthracyclines, it is likely that prospective comparative trials would have been required to demonstrate that adding an anthracycline, with its risk of lifethreatening toxicity, provides a substantial benefit in terms of DFS and OS compared to a non-anthracycline, taxane-based regimen. Instead, taxanes were developed subsequent to anthracyclines 
and as such all early studies compared anthracycline-based regimens to taxane plus anthracycline regimens. In fact, 20 years passed from the publication of the first clinical trial publication of paclitaxel $^{56}$ to the first publication of a head-to-head clinical comparison of a taxane to an anthracycline for early-stage disease ${ }^{57}$.

\section{Head-to-head comparisons of anthracycline vs taxane in the curative setting}

In 1997, Valero and colleagues published promising activity from a phase I study of a new "TC" regimen comprised of docetaxel $\left(75 \mathrm{mg} / \mathrm{m}^{2}\right)$ combined with cyclophosphamide $\left(600 \mathrm{mg} / \mathrm{m}^{2}\right)^{58}$. Stephen Jones, lead author of the original trial of AC for breast cancer in $1975^{17}$ was the first to conduct a formal head-to-head comparison of four cycles of AC vs TC ${ }^{57}$ in 1016 patients with stage I-III breast cancer. Approximately two-thirds of patients had $\mathrm{HR}+$ disease (all of whom received endocrine therapy) and over $50 \%$ of patients had LN involvement (majority $<4$ ). With 5 years follow up, the DFS was significantly higher in patients treated with TC (86 vs $80 \%, p=0.015)^{57}$. This improvement in DFS was seen regardless of age, LN status, or HR status. With 7 years of follow up, OS was significantly improved in patients treated with TC (87 vs $82 \%, p=0.032)^{59}$ regardless of age or HR status. There were four deaths in the $A C$ arm likely related to treatment; one due to CHF and three others due to myelodysplasia (MDS), myelofibrosis, and acute myeloid leukemia (AML). There were no such deaths reported in the TC arm.

A second adjuvant trial, conducted by the Alliance, directly compared a taxane to an anthracycline ${ }^{60}$. In contrast to the US Oncology study ${ }^{57}$, this study utilized single-agent paclitaxel and compared it to the anthracycline doublet, $A C$, and was a noninferiority design ${ }^{60}$. A $2 \times 2$ design was utilized to also compare shorter vs longer therapy (four vs six cycles of $A C$ and 12 vs 18 weeks of paclitaxel). This trial, which was open from 2002-2008, originally aimed to enroll 4646 patients based on $89 \%$ power and 567 RFS events. However, it closed after enrolling 3871 patients. In addition, several changes to the protocol design, including changes to eligibility and treatment regimens, occurred during the study. The original study design utilized standard q3 weekly AC and q-weekly paclitaxel $\left(80 \mathrm{mg} / \mathrm{m}^{2}\right)$. However, in 2003, after 571 patients had been enrolled, both regimens changed to q2 weeks (with paclitaxel dose increased to $175 \mathrm{mg} /$ $\mathrm{m}^{2}$ ). Adjuvant trastuzumab was not incorporated until after 2005. At a median 6.1 years follow up and 437 RFS events (23\% fewer events than required for $89 \%$ power), non-inferiority of paclitaxel could not be concluded with a HR of 1.26 favoring AC. Five-year RFS in AC vs paclitaxel arms was 91 and $88 \%$, respectively. The authors acknowledged the limitations of this study relating to accrual and multiple changes in study protocol, but were reassured by post hoc statistical analyses indicating the overall results would not have been different had full accrual been met, or study design not changed. Fewer than half the patients had HER2 status available and of these, $16 \%$ were HER2+. The multivariable analysis did not include HER2 status. The fact that taxane-treated patients did not receive cyclophosphamide is another potential limitation of this study. No difference in 5-year OS was observed (95\% AC vs $94 \% \mathrm{~T}$ ), in contrast to the Jones study, with the noted major caveat that the Jones study had added cyclophosphamide to the taxane. Importantly, seven patients, all in the AC arm, developed treatment-related AML or MDS, all of whom died.

In addition to the two above adjuvant studies, a phase III neoadjuvant study from the National Cancer Center in Korea directly compared anthracycline to taxane-based therapy. A total of 204 patients with stage II-III breast cancer (all subtypes) were randomized to receive four cycles of q3 weekly docetaxel plus capecitabine $(\mathrm{TX})$ or $\mathrm{AC}^{61}$. After surgery, all patients crossed over to receive four cycles of the other treatment. Pathologic complete response $(p C R)$ in the breast was significantly better in the TX arm (21 vs $10 \%, P=0.024$ ). No difference in DFS was seen, an expected finding given the crossover design of the study.

Results from a substudy of the phase III EORTC 10041/BIG 03-04 (MINDACT) trial has also questioned whether anthracycline-based regimens are needed in the taxane era ${ }^{62}$. In this analysis, 1301 patients with $\mathrm{T} 1-\mathrm{T} 3$ operable tumors and up to three positive nodes were randomized 1:1 to standard anthracycline therapy (70\% anthracycline without taxane, 30\% anthracycline plus taxane) or TX. Patients with HER2+ tumors received standard trastuzumab. Though underpowered to determine superiority for TX, the 5-year DFS was similar for the anthracycline arm (88.8\%) and DX arm (90.7\%) (HR, 0.83; $p=0.26)$ as was the 5 -year OS (96.2\% anthracycline vs $96.3 \%$ DX, HR 0.91, $p=0.72$ ).

\section{Studies comparing taxane/anthracycline vs taxane (in a mixed population of HER2 + and HER2 - disease)}

One of the earliest trials to be published comparing an anthracycline plus taxane to a taxane without an anthracycline was the JCOG9802 trial in which all subtypes of MBC were prospectively randomized to receive six cycles of $A C$, docetaxel, or alternating treatment with $A C$ and $D$ (three cycles of $A C$ followed by three cycles of $D)^{63}$. Patients were eligible if they had taxane naïve, $\mathrm{HR}-$ or endocrine-resistant $\mathrm{HR}+$ breast cancer. Prior adjuvant anthracycline use was allowed. A total of 441 patients were accrued to this multicenter study. Approximately $85 \%$ of patients were anthracycline-naïve. Time to treatment failure (TTF), the primary endpoint, was similar in each of the three arms (6.4 mos AC, 6.4 mos D, 6.7 mos AC-D). OS was 22.4 months in the AC arm, which tended to be worse compared to the D-arms ( 25.7 mos in $\mathrm{D}$ arm, $p=0.09$ and 25.0 mos in AC-D arm, $p=0.08$ ). Responses were observed in $29 \%$ of patients treated with $A C, 40 \%$ treated with $D$, and $35 \%$ treated with ACD. Tumors were not tested for HER2 and trastuzumab was not used. Moreover, the dose of both the anthracycline $\left(40 \mathrm{mg} / \mathrm{m}^{2}\right)$ and taxane $\left(60 \mathrm{mg} / \mathrm{m}^{2}\right)$ used in this trial were somewhat lower than used in the adjuvant setting, possibly impacting results. That said, these data did not suggest any incremental benefit derived from adding an anthracycline to docetaxel in the metastatic setting.

A phase III randomized trial (N-SAS BC 02) conducted at 84 centers in Japan evaluated whether single-agent taxane has a non-inferior DFS compared to anthracycline/taxane-based adjuvant therapy for LN+ breast cancer ${ }^{64}$. From 2000-2006, 1060 patients were randomly assigned to receive every 3 weeks either four cycles of AC followed by four cycles of paclitaxel $\left(175 \mathrm{mg} / \mathrm{m}^{2}\right)$ (ACP), four cycles of AC followed by four cycles of docetaxel (ACD), eight cycles of paclitaxel (P), or eight cycles of docetaxel (D). A $2 \times$ 2 factorial design was utilized to compare AC-taxane-containing regimens ( $A C P$ and $A C D$ ) to taxane without anthracycline ( $P$ and $D)$ and to compare paclitaxel-containing regimens (ACP and $P$ ) to docetaxel containing regimens $(A C D$ and $D$ ). With a median follow up of 7 years, non-inferiority of the single-agent taxane could not be demonstrated (HR 1.19; $90.3 \% \mathrm{Cl}, 1.012-1.405, p=0.30$ ). This finding may have been influenced by the use of $q 3$ weekly $P$ in the two arms which was clearly inferior to $q 3$ weekly D-containing regimens (DFS HR, 0.72; $p=0.0008$ and OS HR 0.75; $p=0.035$ ). Of all four arms, the median DFS was numerically longest in the $D$ arm (ACP: 84.4 mos, ACD: 85.7 months, D: 87.9 mos, and P: 78.0 mos). Interestingly, patients with HER2 + breast cancer (16\% of population, none treated with trastuzumab) did appear to derive greater benefit from the addition of anthracycline to $\mathrm{P}$ (HER2+: ACP vs $P[H R, 1.85 ; 95 \% \mathrm{Cl}, 1.15-2.98])$. 


\section{DOES THE HER2 ALTERATION AFFECT SENSITIVITY TO ANTHRACYCLINES?}

When evidence emerged indicating that HER2 amplification is a poor prognostic indicator in breast cancer ${ }^{65-69}$, investigators began to query whether this outcome may be linked to altered sensitivity to various standard chemotherapies. To this end, archived tumor samples from a number of adjuvant trials conducted in the pre-trastuzumab era were analyzed. Initially, several studies evaluating non-anthracycline-based chemotherapy indicated that HER2 overexpression was associated with resistance to chemotherapy and thus may, at least in part, explain the poor outcome associated with this genetic alteration ${ }^{70-72}$.

Subsequently, a number of investigators evaluated whether the HER2 alteration affects sensitivity to anthracycline-based therapy, using a variety of laboratory methods to evaluate HER2 expression and amplification status. Tumor samples were retrospectively assessed for HER2 status from a total of 12 separate randomized trials $^{73-85}$ comparing non-anthracycline to anthracycline-based adjuvant chemotherapy (Table 1). Of these, three demonstrated a significant interaction between HER2 status and treatment ${ }^{73,76,83}$, indicating that HER2 + breast cancer is associated with a greater benefit in terms of DFS and/or OS from anthracyclines than HER2breast cancer. One of the earliest of these analyses was from the NSABP B11 trial in which patients with $\mathrm{LN}+, \mathrm{HR}$ - disease were randomized to receive $\mathrm{L}$-phenylalanine mustard plus 5-fluorouracil alone or in combination with doxorubicin ${ }^{73}$ Those with HER2 overexpression had significant DFS, RFS, and OS benefit with the addition of doxorubicin. In contrast, those with normal HER2 expression had similar outcomes regardless of anthracycline use. Another large study, the Canadian Mammary.5 (MA.5) ${ }^{83}$ also evaluated HER2 status retrospectively. In this trial, adjuvant CEF was compared to CMF in LN+ disease. Improvements in RFS and OS from anthracycline use were restricted to those patients with HER2 amplified tumors. Subsequently, PAM-50 analysis was performed on these samples, indicating that the HER2-enriched subtype benefited significantly from CEF, however other subtypes, including basal-like breast cancers (frequently TNBC), did just as well with $\mathrm{CMF}^{86}$.

Analysis of another nine trials demonstrated that HER2-positive breast cancers tended to have greater benefit from anthracyclines (in either DFS or OS outcomes) compared to HER2- breast cancers. However, these trends did not reach a statistically significant level of interaction for either outcome measure (Table $1)^{74,77-80,82,85}$. Paik and colleagues analyzed HER2 expression on tumor samples from 1355 of 2194 (62\%) patients enrolled on the NSABP B15 study in which patients with $\mathrm{LN}+$ breast cancer received adjuvant therapy with either $\mathrm{AC}, \mathrm{CMF}$, or $\mathrm{AC} \rightarrow \mathrm{CMF}^{74}$. Outcomes tended to be better in patients with HER2 overexpressing breast cancer treated with AC-based therapy (DFS for $\mathrm{HER} 2+, \mathrm{RR}=0.84$, OS for HER2,$+ \mathrm{RR}=0.82$ ), though this also did not reach statistical significance. In contrast, patients with HER2breast cancers had virtually overlapping outcomes with anthracycline vs non-anthracycline-based therapy, with a slight trend toward better outcomes with CMF treatment (DFS HER2 - RR = 1.02; OSHER2 $-R R=1.07)$. Similarly, HER2 analysis $(N=506)$ from a study in Milan ${ }^{79}$ demonstrated a strong trend toward survival benefit with anthracyclines only in those with HER2overexpressing disease (OS CMF-A vs CMF HER2+: HR $=0.61$, HER2 - HR:1.26; $p$ interaction $=0.052$ ). Likewise, analysis of samples $(N=481)$ from a Belgian study ${ }^{77}$ comparing CMF to high dose EC (HEC, epirubicin at $100 \mathrm{mg} / \mathrm{m}^{2}$ ) indicated a strong trend toward improved event-free survival (EFS) for those patients with HER2 overexpressing breast cancer (HR: 0.33) whereas those with HER2 - breast cancer seemed to derive just as much benefit from CMF (HR:1.16). While analysis from the BR9601 study $(N=303)^{84}$ showed no significant interaction between treatment, HER2 status, and outcome, samples were tested from tissue microarrays (TMA) which, according to one study, may alter the reliability of HER2 result ${ }^{87}$. When the BR9601 samples were combined with samples from the similarly designed NEAT trial ${ }^{85}$, the sample size increased to 1762 , and again a trend toward improved OS was noted in favor of anthracycline-based therapy for HER2+ $(H R=0.74)$ compared to HER2 - disease $(\mathrm{HR}=0.84$; total HR $0.81 ; p=0.02$; $p$ interaction $=0.55$ ).

DBCG 89d, which evaluated samples from 805 patients (67\%) randomized to CEF vs CMF, showed no difference in EFS when comparing hazard ratios for HER2+ and HER2- disease ${ }^{81}$. However, a recently published analysis ${ }^{88}$ of 686 samples from this study using the Prosigna Prognostic Gene Signature indicates that patients with the HER2-enriched subtype have a better distant relapse and OS when treated with CEF vs CMF. The same was not observed in the other intrinsic subtypes. Discordance between HER2-enriched status and HER2 FISH status was noted. Of 217 patients with HER2-enriched tumors, 32 (15\%) were HER2- by FISH. Only those that were HER2-enriched appeared to benefit from epirubicin. Of 469 classified as non-HER2-enriched, 38 (8\%) were HER2-amplified by FISH but did not show benefit with epirubicin, though the sample size was quite small.

Given these somewhat conflicting results, from trials using different methods to define HER2 status, and some trials lacking sufficient statistical power to critically analyze efficacy endpoints in the defined HER2 + subset, Gennari and colleagues performed a pooled analysis of the interaction between HER2 and anthracycline benefit ${ }^{89}$. Eight studies (Table 2) including over 5000 patients comparing adjuvant anthracycline to non-anthracycline chemo were analyzed. Methods for defining HER2 status differed across studies, as indicated above and in Table 1, and included IHC, FISH, and polymerase chain reaction. In spite of this heterogeneity, this meta-analysis, with its greatly increased power, clearly demonstrated that anthracycline benefit was restricted to those patients with HER2 + disease. HER2 + breast cancers had a $29 \%$ relative risk reduction for DFS events and $27 \%$ relative improvement in survival with the use of anthracycline. Conversely, there was no incremental benefit from an anthracycline vs a non-anthracycline regimen in HER2- disease. The interaction for both DFS and OS was statistically significant ( $p<0.001$ for both). It is notable that when HER2 + and HER2 - breast cancers were analyzed together as a single group, the combined DFS (HR 0.90) and OS (HR 0.91) were better with the anthracycline regimens. However, in sensitivity analysis, this benefit is completely lost when the HER2 + breast cancers are removed from the meta-analysis. This relative benefit seen in the combined population is strikingly similar to that reported from the EBCTCG meta-analysis ${ }^{3,41}$ in which a slight improvement in DFS and OS was seen with the use of anthracyclines in a mixed pool of breast cancer patients unselected for HER2 status. Subsequently, a pooled analysis ${ }^{90}$ of these studies plus one other study ${ }^{76}$ confirmed the findings of the Gennari analysis (Table 2).

In 2011, Di Leo and colleagues published another analysis of five studies using individual patient data (Table 2$)^{87}$. Unique to this was the fact that the authors attempted to independently assess HER2 status in a centralized laboratory located in Tampere, Finland. However, when high rates of discordance were detected between the external laboratory in Tampere and the four national laboratories that tested the samples from each of the trials, it was decided that the use of TMA might be to blame and only a portion of patient samples $(N=137)$ from whole tumor sections were sent for repeat HER2 FISH testing at the external central lab. The concordance rate for HER2 results between the national laboratories and the external central laboratory for these samples was $94 \%$. Four of the included studies ${ }^{78,81,84,85}$ had failed to demonstrate a significant association between HER2 status and outcome with anthracycline-based therapy. However, when analyzed together with over 3400 samples and 1417 events, the EFS was significantly in favor of anthracyclines for HER2+ disease 


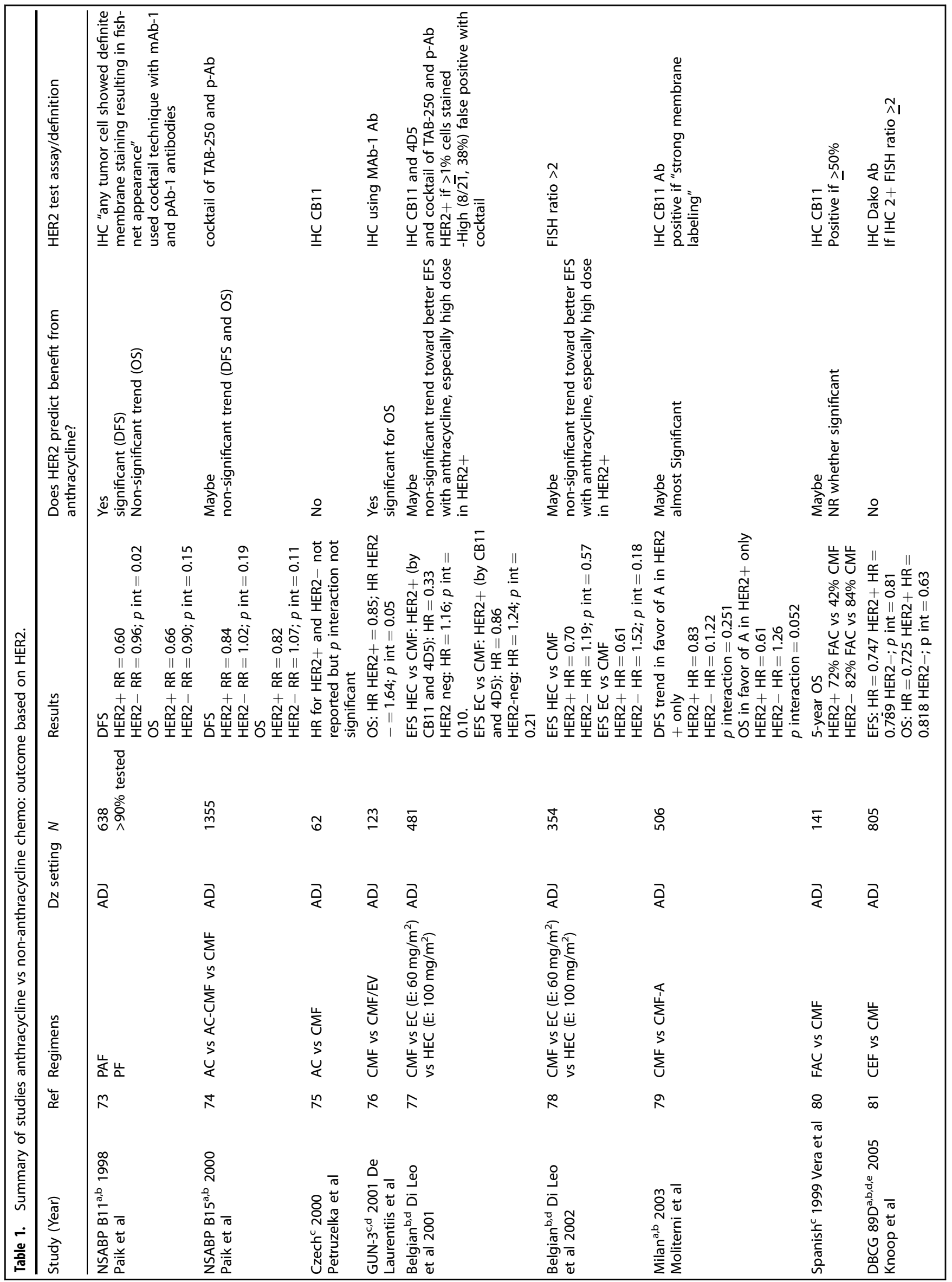




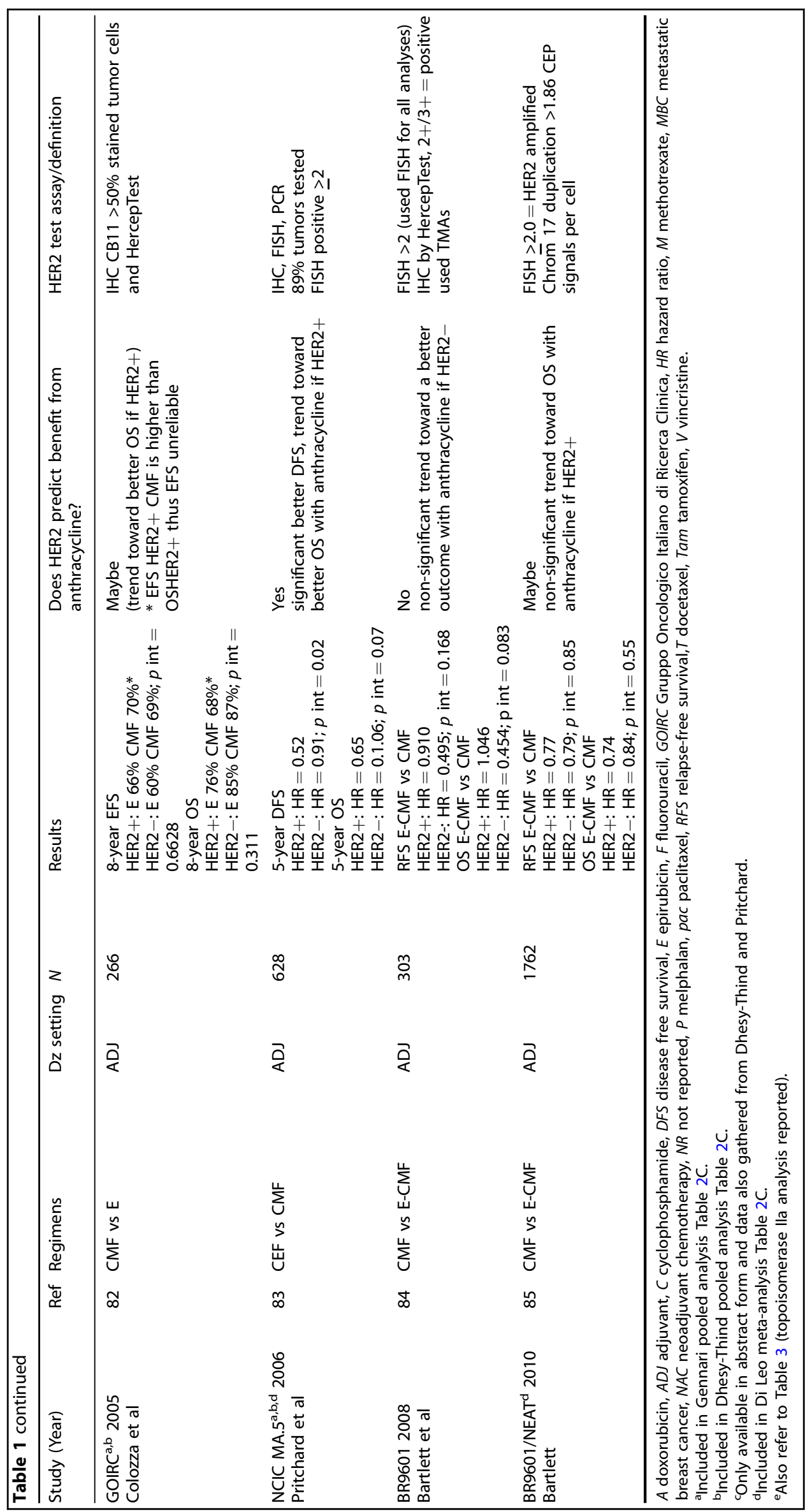




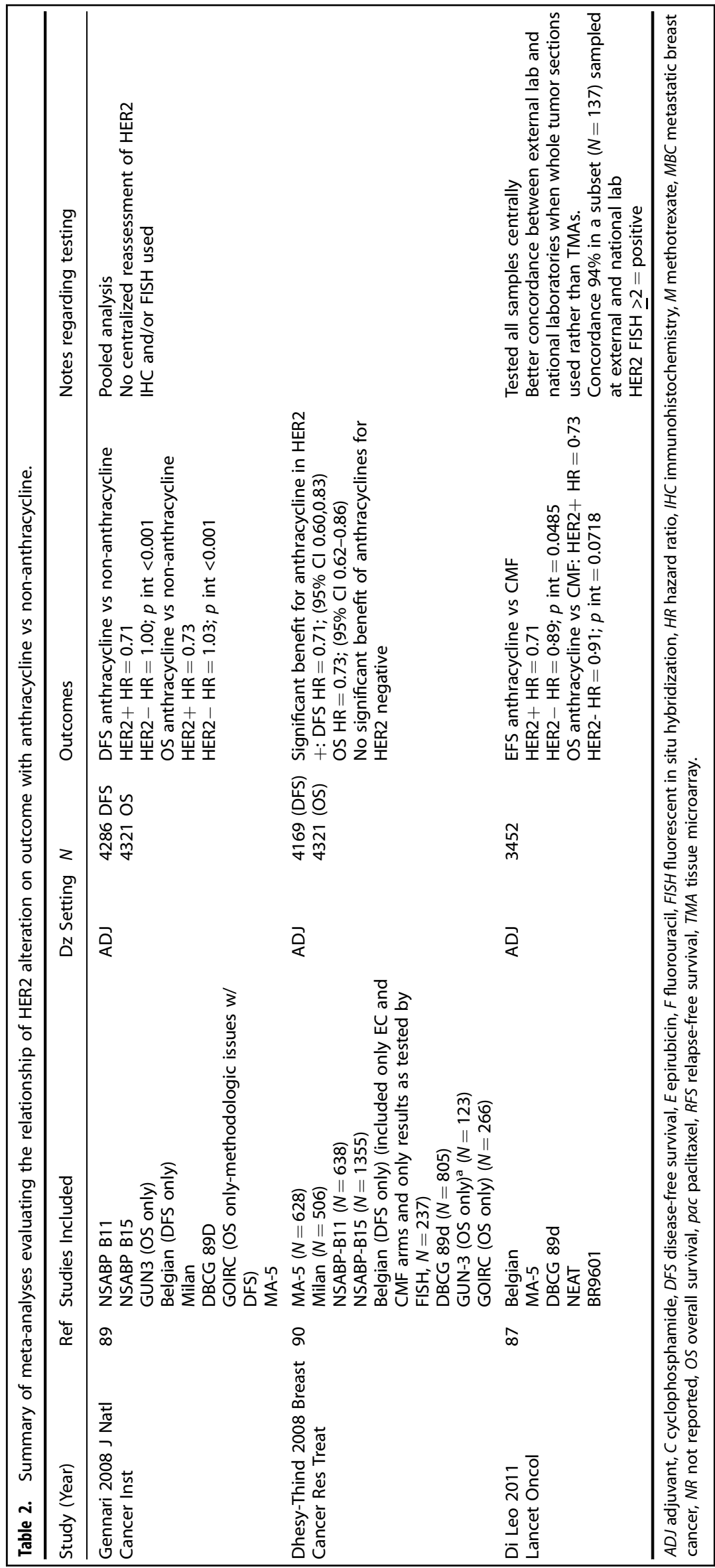


$(\mathrm{HR}=0.71)$ compared to $\mathrm{HR}=0.89$ for HER2 - breast cancer ( $p$ interaction $=0.0485$ ). OS also trended toward an improved benefit with anthracyclines for HER2 + breast cancer ( $\mathrm{HR}=0.73)$ compared to HER2 - $(\mathrm{HR}=0.91)$.

A handful of other trials evaluated whether patients with HER2+ breast cancer receive greater benefit from dose intense anthracyclines, defined as either a higher cumulative dose or more frequent dosing in the curative setting (Table 3). One of the first of these analyses was conducted by Muss and colleagues and demonstrated a clear dose-response relationship with doxorubicin in patients with HER2 + cancers, but not those with HER2 - disease ${ }^{91-93}$. In their initial analysis, samples from 397 patients treated in the CALGB 8541 study were analyzed for HER2 by IHC. In both the overall study and in the subset of patients included in this biomarker analysis, DFS and OS were improved in patients receiving higher doses of chemotherapy (groups 1 and 2). In those with HER2+ tumors, DFS and OS were significantly associated with a higher dose of chemotherapy. Focusing on all patients treated with high-dose chemotherapy, those with HER2 + tumors had a better DFS and OS compared to patients whose tumors were HER2 $-{ }^{91}$. Tumor samples from an additional 595 patients were subsequently analyzed along with the original 397 samples $^{92}$. In the group of 397 patients with a median follow up of 10.4 years, the association between HER2 and anthracycline dose was even stronger than reported in the initial analysis, though this dose-response relationship in HER2 + breast cancer did not appear to hold for doses of doxorubicin above $60 \mathrm{mg} / \mathrm{m}^{2}$. Indeed, similarly, an analysis of patients treated on the CALGB 9344 study $^{94}$ in which patients were assigned to 60, 75, or $90 \mathrm{mg} / \mathrm{m}^{2}$ showed a comparable 5-year DFS irrespective of HER2 status.

Several additional studies ${ }^{77,78,95-98}$ also showed a trend toward a positive relationship between the HER2 alteration and benefit from dose intense anthracyclines. Dhesy-Thind ${ }^{90}$ performed a meta-analysis ${ }^{78,92,96}$, using FISH data from the Belgian study ${ }^{78}$ and data from the high dose and medium dose anthracycline arms from the CALGB 8541 analysis ${ }^{91}$. This showed that those with HER2 + breast cancer derive a significant DFS benefit from dose intense chemotherapy $(\mathrm{HR}=0.54,95 \% \mathrm{Cl} 0.38-0.79)$ whereas those with HER2- disease do not $(\mathrm{HR}=0.98)$. While two studies $^{99,100}$ failed to show an association with HER2 status and benefit from an additional cycle of anthracycline, these trials were confounded by the addition of either taxane ${ }^{99}$ or high dose chemotherapy followed by stem cell rescue ${ }^{100}$ in the arm with less anthracycline.

A meta-analysis conducted by the EBCTCG evaluated the benefits of giving chemotherapy drugs either more frequently or sequentially (instead of concurrently) in early-stage disease ${ }^{101}$. Dose intensification provided a modest benefit in recurrence risk for patients regardless of HER2 status. However, all arms of the included studies were treated with an anthracycline and the majority also received a taxane. Thus, this analysis was not designed to specifically address whether higher cumulative anthracycline doses are associated with benefit based on HER2 status and this analysis does not directly bear upon the question as to whether the addition of anthracyclines per se provides benefit.

Taken together, these data provided a credible link between the HER2 alteration and sensitivity to anthracycline-based therapy. Indeed, the first two FDA approvals for HER2 FISH testing were to risk stratify patients ${ }^{102}$ and select those who were at greater risk for recurrent disease ${ }^{103}$ and/or death and to select those who might benefit from anthracycline chemotherapy (https://fda. report/PMA/P980024S001) ${ }^{104}$. In spite of the circumstantial evidence supporting a link between the HER2 alteration and anthracycline efficacy, it was not clear whether HER2 was causal in heightened anthracycline sensitivity or if it was simply a surrogate marker for another molecular alteration that explained increased responsiveness to anthracyclines. To evaluate this, MCF7 cells were transfected with HER2 CDNA to be rendered HER2 overexpressing and then treated with tamoxifen or chemotherapy. These cells proved resistant to tamoxifen both in vitro and in vivo, however, their sensitivity to anthracycline chemotherapy was unchanged despite HER2 overexpression ${ }^{105}$. Similarly, Pegram and colleagues transfected full-length human HER2/neu CDNA into MCF7, MDA-MB-231, MDA-MB-435, and BT-20 breast cancer cell lines and two ovarian cancer cell lines and compared the response of these cell lines in vitro and in vivo to that of mock-transfected parental lines ${ }^{106}$. These experiments also failed to demonstrate an association between HER2 overexpression and sensitivity or resistance to chemotherapy, including doxorubicin. Orr and colleagues transfected normal human mammary epithelial cells with HER2 and determined sensitivity to multiple chemotherapy agents and also showed that HER2 overexpression is not associated with differential sensitivity to chemotherapy ${ }^{107}$. Further data was provided from Konecny et al. from an in vitro study analyzed at UCLA in which breast cancer samples from 140 chemotherapy-naïve patients from the University of Munich, Klinikum Grosshadern, taken at the time of surgery were treated with either CMF or FEC at different concentrations ex vivo ${ }^{108}$. This, too, showed no association between HER2 overexpression and resistance to either regimen. Before considering possible alternative reasons for differential anthracycline sensitivity being observed clinically in HER2 + disease, data relating to the benefits of adding anthracyclines to taxanes in HER2 negative disease will be explored.

\section{PROSPECTIVE STUDIES DESIGNED TO EVALUATE TAXANE/ ANTHRACYCLINE VS TAXANE ONLY IN HER2 NEGATIVE EARLY- STAGE DISEASE}

Although the above retrospective analyses did not consistently support the restricted use of anthracyclines to HER2+ breast cancers, the data clearly indicated that benefit from anthracyclines appeared to be limited, at best, in HER2 - disease. Moreover, none of the above studies (Tables 1 and 2) involved the use of a taxane. Thus, a number of investigators aimed to prospectively evaluate whether the addition of anthracyclines to modern taxane-based regimens was associated with a meaningful benefit in patients with curable HER2 - disease. In 2007, US Oncology Research initiated a randomized trial (USOR 06-090) designed to evaluate whether six cycles of docetaxel/doxorubicin/cyclophosphamide (TAC) is superior to six cycles of TC in LN positive or high-risk node-negative breast cancer. A second study, developed jointly by the NSABP and USOR was then initiated in 2009 to evaluate three arms: TCx6, TACx6, and TC plus bevacizumab $\times 6$ with a planned enrollment of 3600 patients. The plan was to combine the TC and TAC arms from the USOR 06-090 and NSABP B-46-I/USOR 07132 studies. However, given the withdrawal of FDA approval of bevacizumab, it was decided to close this study after enrollment of only 1077 patients in early 2012. To convert the study to a non-inferiority design, a third study (NSABP B-49) was opened in 2012. In this trial, patients were randomized to TC or to four different anthracycline/taxane-based regimens. This study enrolled 1870 patients. The joint analysis of these three trials (collectively known as "ABC") was published in $2017^{109}$ and marks the first to address whether a non-anthracycline, taxane-based regimen is non-inferior to the anthracycline/taxane regimens in terms of invasive DFS (iDFS). Of 4156 patients, 31\% had TNBC, 59\% had $\mathrm{LN}+$ disease, and $51 \%$ had high-grade tumors. An interim analysis with a median follow up of 3.3 years and 399 observed iDFS events, demonstrated the observed HR was $1.23(95 \% \mathrm{Cl} 1.01$, $1.50 ; p=0.04)$, thus non-inferiority could not be concluded. The absolute difference between treatment arms in 4-year iDFS was 2.5\% (TC: $88.2 \%$, TaxAC: $90.7 \%$ ). Most of the benefit for the anthracycline arms was observed in those with four or more LN involved. In that group, the projected absolute difference between 


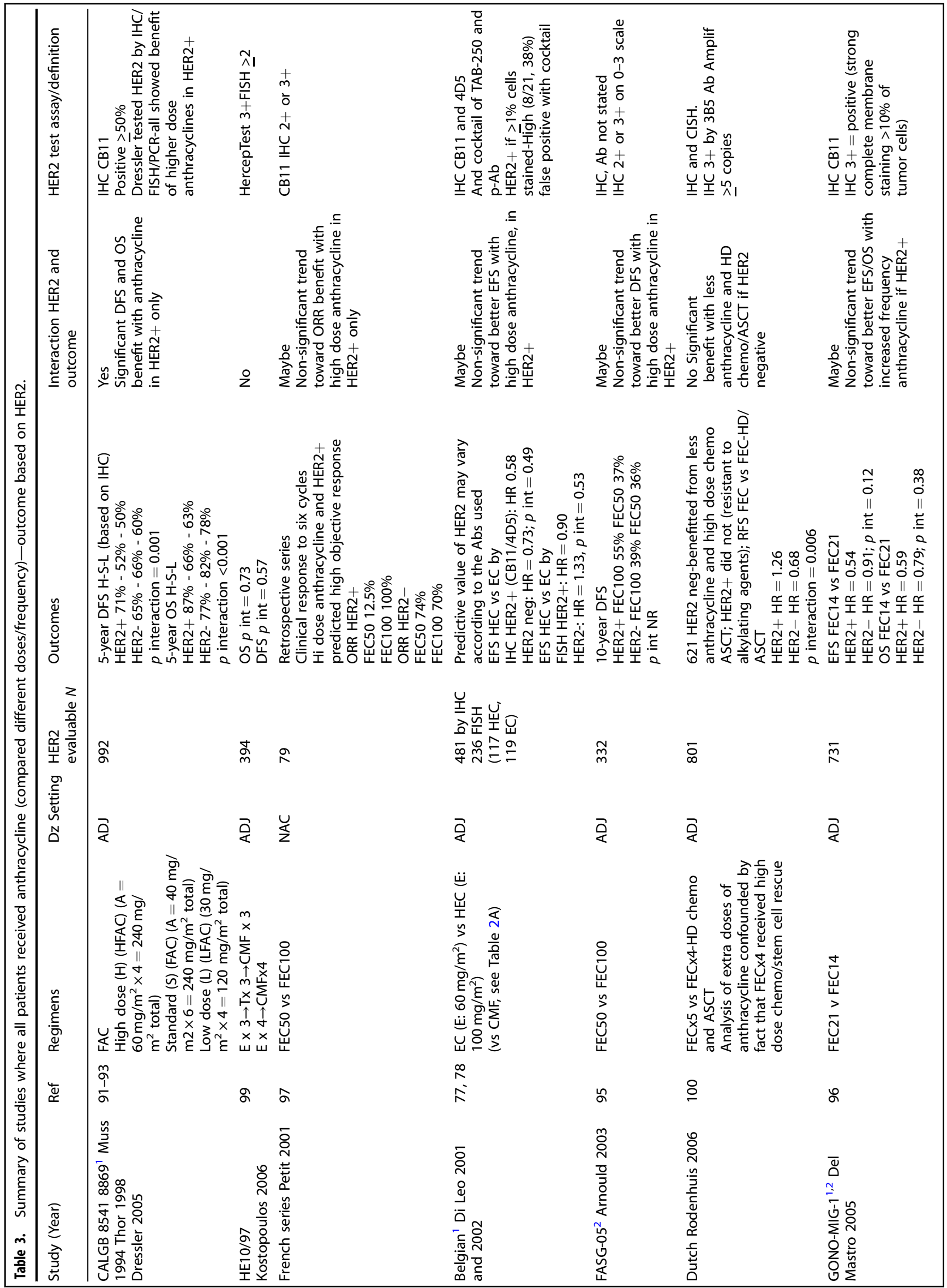


the two arms (by Kaplan-Meier analysis at 4 years) was $11 \%$ for TNBC and $5.8 \%$ for $\mathrm{HR}+$. In the overall population, distant recurrences were observed in $5.3 \%$ of TC-patients vs $3.6 \%$ of anthracycline-patients. Deaths rates were similar in each arm (1.1\% TC and $1.4 \%$ anthracycline). There were five acute leukemias diagnosed in anthracycline-treated patients, none in TC-treated patients. Notably, toxicity data was only published in the main manuscript for the NSABP B-49 trial. Safety data from the other two studies appear in the Data Supplement. No grade $\geq 3$ cardiomyopathy was reported for TC however at least three patients died from cardiomyopathy or heart failure in the anthracycline arms, per the Supplementary Data. It is also notable that cardiac function was not measured throughout the study so occult cardiac dysfunction rates are not known.

Given the lack of centralized pathology review and the use of a HER2/CEP17 ratio of $<2.2$ (rather than the currently FDA approved $<2.0$ ), it is plausible that some patients with HER2 amplified breast cancer may have been enrolled. Whether this is the case and whether or not this impacted the iDFS results remains unknown. As of this time, no updates or biomarker analyses from this study have been presented or published.

Subsequently, a number of prospectively designed trials to address this issue have been reported, demonstrating no significant benefit with the addition of an anthracycline to taxane therapy. The West German Study Group phase III PlanB study successfully demonstrated the non-inferiority of a nonanthracycline, taxane-regimen ${ }^{110}$. This prospective, randomized study evaluated whether six cycles of adjuvant TC is non-inferior to four cycles of EC followed by four cycles of docetaxel $(100 \mathrm{mg} /$ $\mathrm{m} 2)$ in patients with HER2 - breast cancer $(N=2449)$. Forty percent of patients had $\mathrm{LN}+$ disease, $17 \%$ had centrally confirmed TNBC, and $42 \%$ had high-grade tumors. With a median follow up of 60 months, the 5-year DFS was $89.6 \%$ for EC-T vs $89.9 \%$ for TC (TC vs EC-T HR $=0.996$ ) and within the noninferiority margin. In contrast to the $A B C$ study, subset analysis indicated a similar DFS in each treatment arm regardless of recurrence score, LN status, grade, or TNBC subtype.

Another study, SUCCESS-C, was also undertaken to prospectively evaluate this question. In this 3642-patient phase III trial, treatment with three cycles of FEC followed by three cycles of $D$ was compared to TC for six cycles. A pooled analysis of this trial with the results of PlanB (above) were presented in $2018^{111}$. Of 5923 patients included, 2979 were assigned to non-anthracyline and 2944 to anthracycline; $52 \%$ had LN+ disease, $40 \%$ had grade 3 disease, and $22 \%$ had TNBC. With 62 months median follow up, DFS for the two arms were almost identical (HR 1.04, $p=0.64$ ) and remained similar regardless of luminal subtypes or triplenegative status. The only group that appeared to benefit from anthracycline-based therapy were those with four or more involved LN (DFS $75 \%$ for non-anthracycline and $82 \%$ for anthracycline).

A smaller $(N=650)$ phase III, a non-inferiority study run by the Hellenic Oncology Research Group (HORG) evaluated 3-year DFS of dose-dense FEC-D (eight cycles total) vs six cycles of TC in women with LN positive disease ${ }^{112}$. Notably, over one-third of patients had at least four LN involved. The 3-year DFS was $89.5 \%$ with FEC-D and 91.1\% with TC (HR 1.147, $p=0.568)$, though noninferiority was not met.

Taken as a whole, these studies indicate the benefit of adding an anthracycline to taxane-based chemotherapy in HER2disease appears to be marginal at best, especially in HR+ disease, and likely is restricted to patients with four or more LN involved.

\section{Studies comparing taxane/anthracycline vs taxane exclusively in TNBC}

Given the poor prognosis associated with TNBC and the lack of targeted systemic therapy options, the choice of a chemotherapy 
regimen to reduce the risk of distant recurrence may be of particular importance. It is interesting to note that early analyses in the pre-taxane era did not clearly indicate the TNBC subtype uniquely benefits from anthracyclines. For example, a Korean registry analysis ${ }^{13}$ of 4033 patients who had node-negative, triple-negative breast cancer treated with CMF (29.5\%), AC $(35.2 \%)$, FAC $(21.7 \%)$, or no chemotherapy were evaluated for survival outcomes. While receipt of chemotherapy was significantly associated with improved OS compared to no chemotherapy, there was no difference in survival when comparing the three adjuvant regimens to one another. Similarly, an exploratory analysis of the MA- 5 trial evaluating outcomes based on PAM-50 intrinsic subtypes indicated basal-like breast cancer does not benefit from anthracycline and may in fact benefit more from CMF chemotherapy ${ }^{86}$. In contrast, subgroup analysis of the ABC trial in which taxane combinations were utilized ${ }^{109}$ appears to indicate that TNBC derives more benefit from an anthracycline (TC vs TAC TNBC HR 1.42; hormone receptor+ HR 1.12) and is most apparent in TNBC with nodal involvement.

In the past several years, encouraging results have been reported for anthracycline-free, taxane plus platinum chemotherapy regimens for early-stage TNBC. Combined results from two prospective cohorts (University of Kansas and Spain) including 190 patients with TNBC, more than half of whom had LN + disease, treated with six cycles of neoadjuvant docetaxel and carboplatin (TP) reported ${ }^{114}$ a pCR rate of $55 \%$ and Residual Cancer Burden (RCB) $0+1$ (pCR plus near $\mathrm{pCR}$ ) rate of $68 \%$. Rates were similar regardless of $B R C A$ mutation status. The estimated 3-year RFS were $79 \%$ and 3 -year OS was $87 \%^{115}$. Importantly, the 3 -year RFS and OS for those who achieved pCR were 90 and 94\%, respectively. Use of adjuvant anthracycline-based chemotherapy was rare in those with pCR (5/100). Nearly $60 \%$ of patients with the significant residual disease received anthracyclines postoperatively but this was not associated with a difference in RFS or OS.

A single-arm phase II Peruvian study evaluated $\mathrm{pCR}$ rates associated with TP for six cycles $(N=27)$ vs historical controls treated with standard $A C$ for four cycles followed by 12 weekly doses of paclitaxel $(N=34)$ for high-risk TNBC ${ }^{116}$ Over threequarters had LN involvement and those treated with TP had a significantly larger median tumor size (72.8 vs $52.2 \mathrm{~mm}, p=0.007$ ). Despite this, the pCR rate was $37 \%$ in the TP arm and $23.5 \%$ in the AC-T arm and 2-year DFS (73.1 vs $59.3 \%$ ) and OS (84 vs $71 \%$ ) were numerically higher with TP.

To date there have been at least six prospective randomized phase II or III clinical trials, three adjuvant and three neoadjuvant (including one led by Sharma and colleagues) ${ }^{117}$, comparing an anthracycline-free, taxane/platinum regimen to a taxane/anthracycline-based regimen in early-stage TNBC (Table 4). All demonstrated either similar or improved outcomes with the nonanthracycline-based regimen ${ }^{17-122}$. Though each of these individual trials was relatively small in size, as a whole they provide data from a total of 828 patients with TNBC, failing to demonstrate that this disease subtype derives a significantly greater benefit from the use of an anthracycline/taxane vs a taxane/platinum-based, non-anthracycline regimen. However, the fact that the TNBC subtype, which accounts for only $10-15 \%$ of breast cancer, is comprised of molecularly heterogeneous subtypes makes interpreting efficacy outcomes with cytotoxic chemotherapy in this group of tumors even more challenging.

\section{SELECTING PATIENTS FOR ANTHRACYCLINES: TOPOISOMERASE II ALTERATION}

Given the differential data regarding the benefit of anthracyclines in both HER2 - and HER2 + breast cancer as well as the clear preclinical data showing that transfection of the HER2 gene itself does not impart sensitivity to anthracyclines, the search for predictive biomarkers continues. Topoisomerase II alpha (TOP2A) codes for a critical enzyme involved in DNA replication ${ }^{123,124}$ and has been identified as a candidate gene for three reasons. First, it is located on the long arm of chromosome 17 in relatively close proximity to the HER2 locus and sometimes is co-amplified with $H E R 2$. Second, its protein product (Topolla) is a direct target of anthracycline chemotherapy. Finally, TOP2A amplification has been shown preclinically to be associated with increased protein expression and increased sensitivity to anthracycline chemotherapy ${ }^{123,125,126}$ Conversely, deletion of TOP2A has been associated with decreased expression of Topolla and resistance to anthracycline ${ }^{125,127}$.

Before discussing the data relating to topoisomerase amplification/expression with response to anthracyclines, it is important to review evidence relating to the incidence of TOP $2 A$ amplification in relation to HER2 amplification, and to highlight how both testing techniques and result interpretation for topoisomerase have varied, leading many to draw conflicting and confusing conclusions.

\section{TOP2A amplification and relation to HER2 amplification}

Interpretation of a number of studies evaluating HER2 and TOP2A amplification have been complicated by the definitions used for amplification. For example, two early studies evaluating HER2 and TOP2A alterations by FISH in breast tumors $\left(N=136^{128}\right.$ and $N=$ $97^{125}$ ) indicated that over $40 \%$ of HER2 amplified breast cancers have TOP $2 A$ co-amplification. This high incidence may be related to the fact that authors defined amplification for both genes as a copy number ratio of $\geq 1.5$, rather than $2.0^{125}$. In HER2 normal tumors, no TOP2A alterations were detected. An analysis of samples from the BR9601/NEAT ${ }^{84}$ demonstrated 9 of 26 TOP2Aamplified tumors were HER2-normal by FISH. However, again the cutoff ratio set for TOP2A amplification was $>1.5$ so tumors that were not actually amplified (defined as $>2.0$ ) were likely included. Similarly, two Polish series indicated amplification of TOP $2 A$ occurs in a substantial proportion of HER2 non-amplified cancer, however again the definition of amplification (TOP2A/CEP17>1.25) undoubtedly led to the counting of TOP $2 A$ non-amplified tumors as amplified ${ }^{129,130}$.

At least ten other studies evaluating alterations in these two genes demonstrated that TOP $2 A$ is only amplified in the presence of HER2 amplification ${ }^{131-140}$ and an additional 12 studies reported TOP2A amplification in very few cases without HER2 amplification $^{141-153}$. Taken together, the incidence of HER2/TOP2A coamplification from these studies was roughly $35 \%$. The largest of these analyses evaluated 4943 breast cancers, all tested by FISH in one academic central laboratory (USC, Los Angeles, CA, USA) using methods and probes validated by the physical mapping on the 17q12-q21 amplicon ${ }^{137}$. Both HER2 and TOP2A amplification were defined as a copy number ratio to centromere 17 (CEP17) of $\geq 2.0$. The test set consisted of 339 tumors from patients with $M B C$ treated on the registrational trastuzumab (H0648) trial. Of these, 279 were confirmed to be $H E R 2$ positive by FISH, 99 of which were TOP2A co-amplified (35\%). No TOP2A amplification was observed in HER2 normal tumors. An additional 4604 tumors from the Breast Cancer International Research Group (BCIRG005, $N=1614$ and BCIRG006, $N=2990$ ) trials served as the validation cohort. Again, all tumors were confirmed to be HER2-non-amplified (BCIRG005) or HER2-amplified (BCIRG006) by FISH in the same central laboratory, using validated probes and methods as well as the FDA approved cutoff for HER2 amplification. Amplification of TOP2A was detected in 35\% (1057/2990) of HER2-amplified tumors. Not a single case of TOP2A amplification was detected in 1614 HER2 normal tumors.

In addition to differing cutoffs for defining amplification, other factors may account for the fact that some studies have reported TOP2A amplification in HER2 normal tumors including the source of tissue for testing, differing assays (qPCR vs FISH), and lab-to-lab 
Table 4. Prospective randomized trials of taxane/platinum vs taxane/anthracycline in early triple-negative breast cancer.

\begin{tabular}{|c|c|c|c|c|c|c|}
\hline Study (Author/Year) & Ref & Treatment & Dz Setting & Phase & $N$ & Outcomes \\
\hline Wang J 2019 (abstract) & 118 & $\begin{array}{l}\text { Dose-dense paclitaxel/carboplatin vs Dose-dense } \\
\mathrm{EC} \rightarrow \text { paclitaxel }\end{array}$ & ADJ & III & 132 & $\begin{array}{l}\text { 3-year DFS } \\
\text { non-anthracycline: } 94 \% \\
\text { anthracycline: } 78 \%, \mathrm{HR}=0.305 \text {, } \\
p=0.0046 \\
\text { 3-year OS } \\
\text { non-anthracycline: } 98 \% \\
\text { anthracycline: } 93 \%, p=0.0268\end{array}$ \\
\hline Du F 2020 (published) & 120 & $\begin{array}{l}\text { Six cycles q3w } \\
\text { Carboplatin AUC } 5 \\
\text { Docetaxel } 75 \mathrm{mg} / \mathrm{m}^{2} \text { or paclitaxel } 175 \mathrm{mg} / \mathrm{m}^{2} \text { vs four } \\
\left.\text { cycles EC (epi: } 90 \mathrm{mg} / \mathrm{m}^{2}\right) \rightarrow \text { docetaxel }\left(75 \mathrm{mg} / \mathrm{m}^{2}\right) \text { or } \\
\text { paclitaxel }\left(175 \mathrm{mg} / \mathrm{m}^{2}\right)\end{array}$ & ADJ & II & 308 & $\begin{array}{l}\text { Median follow up } 66.9 \text { months } \\
\text { 5-year DFS } \\
\text { non-anthracycline: } 84.4 \% \\
\text { anthracycline: } 85.8 \% p=0.712 \\
\text { 5-year OS } \\
\text { non-anthracycline: } 93.5 \% \\
\text { anthracycline: } 94.4 \%, p=0.770\end{array}$ \\
\hline
\end{tabular}

A doxorubicin, $A D J$ adjuvant, $A U C$ area under the curve, $C$ cyclophosphamide, $D F S$ disease-free survival, $E$ epirubicin, $H R$ hazard ratio, $M B C$ metastatic breast cancer, $N A C$ neoadjuvant chemotherapy, $p C R$ pathologic complete response, $O S$ overall survival, $R C B$ residual cancer burden index, RFS relapse-free survival.

variations leading to discordant results. One example that highlights these issues is a meta-analysis of individual patient data from five studies where TOP2A was evaluated. Samples from the Belgian trial, MA-5, DBCG 89D, BR9601, and NEAT trials were included $^{87}$. It is notable that in the original analysis of the Belgian trial $^{78}, T O P 2 A$ amplification was defined as a copy number ratio of at least 1.5 and in the BR9601 and NEAT trials ${ }^{84,85}$ it was defined as a copy number ratio of $>1.5$. In this meta-analysis, however, the definition for TOP $2 A$ amplification changed to $\geq 2$ without an explanation to support the use of either 1.5 or 2 . The investigators originally planned to have all tumors from these five trials retested for TOP $2 A$ and HER2 centrally at an external laboratory (University of Tampere, Finland). However, discordance in results from Tampere and the four national laboratories that performed the original analyses for these five trials was noted and thought to be due to the use of tumor sections cut from TMA. When the external laboratory at Tampere used whole tumor sections, the concordance rate improved. In the end, only a handful $(123 / 3,102$, $3.9 \%$ ) of samples were tested for TOP2A in Tampere and the final concordance between the central lab and the national laboratory was only $69 \%$.

\section{Measuring topoisomerase expression}

While early evidence suggested amplification of TOP $2 A$ is associated with overexpression of the protein ${ }^{125}$, differentiating overexpression from normal expression in tumor samples has been challenging and demonstrating a correlation between amplification and expression has not yielded consistent results. It is now well recognized Topolla is highly expressed in rapidly dividing, high-grade tumors and can thus be a marker of proliferation rate ${ }^{154-160}$. This is consistent with the critical role played by Topolla in cell division. Topolla is known to be a tightly regulated gene at both the transcriptional and translational levels whose expression varies dramatically during the cell cycle. The variability in expression throughout the cell cycle likely accounts for the lack of clear correlation between gene copy number and protein level ${ }^{161,162}$. For example, a Canadian group evaluating HER2 and 
TOP2A by FISH and their protein products by IHC in 81 breast tumor samples observed no correlation between TOP2A-amplification and Topolla protein expression ${ }^{145}$. Other groups also demonstrated a poor correlation between TOP2A gene amplification and Topolla protein expression ${ }^{130,149,153,158,159,163,164}$. Irrespective of this inherent molecular variability, a number of investigators evaluated tumors for Topolla overexpression, using a variety of definitions, in order to assess for a correlation with outcome in anthracycline-treated patients ${ }^{77,84,131,133,136,138,139,165-170}$. Given the lack of standardization of interpreting IHC results for Topolla, the nonexistence of a clear correlation between amplification and expression and evidence to suggest that outcome with anthracyclines is associated with amplification, not expression level ${ }^{136}$, the below section will exclude those studies where topoisomerase was only evaluated by $\mathrm{IHC}^{165-167}$ or mRNA ${ }^{171}$, rather than FISH.

\section{TOP2A amplification and response to anthracyclines}

Numerous retrospective analyses (Tables 5 and 6) have been conducted to evaluate whether TOP2A amplification is associated with response to anthracyclines. While the majority have indicated either a significant association or a trend between TOP2A amplification and benefit from anthracycline, a handful have not. As a whole, the results are difficult to interpret due to their retrospective nature, generally small sample sizes as well as their non-standardized testing techniques and varying definitions of alterations.

Six studies in the neoadjuvant ${ }^{131,136,152,172-174}$ setting have suggested TOP $2 A$ amplification is associated with better pathologic response to anthracycline-based therapy. Analysis of samples from the adjuvant Scandinavian Breast Group 9401 trial $^{175}$ suggested that patients with TOP2A amplified tumors $(N=48)$ had a better RFS when treated with dose escalated FEC compared with standard FEC followed by high dose chemo and stem cell rescue $(\mathrm{HR}=0.45, p=0.049)$. There was no difference in outcome between the two treatment arms in the TOP2A normal group. In contrast, analysis of the CALGB 8541 trial $^{144}$, in which patients were assigned to low, moderate, or high (now standard) dose anthracycline, failed to demonstrate an association with TOP2Aamplification and benefit from high dose CAF. Only 41 patients spread across three treatment arms had TOP2A-amplified tumors and no information was provided regarding how many patients were assigned to each dose level. Therefore, this analysis was conspicuously underpowered. Other retrospective analyses of adjuvant studies in which all patients received the same dose of anthracycline have also been conducted with varying results ${ }^{139,143,148}$.

Press and colleagues analyzed TOP2A status on 279 HER2 amplified tumors from the H0648 trial in which patients were randomized to receive chemotherapy (either $A C$ or paclitaxel) alone or with trastuzumab ${ }^{137}$. They demonstrated a significant association between

TOP2A co-amplification and improved survival $(p=0.004)$ in patients treated with an anthracycline. No difference in survival was noted in patients with TOP2A amplified or non-amplified tumors who were treated with paclitaxel.

Five larger randomized studies comparing anthracycline vs nonanthracycline chemotherapy were also analyzed retrospectively for TOP2A. Di Leo and colleagues analyzed samples from the Belgian study ${ }^{77,78}$ comparing adjuvant HEC to either standard dose EC or to CMF. Benefit with HEC vs CMF appeared to be restricted to those with Topolla expression (HR 0.66) compared to those without expression (HR 1.26), ( $p$ interaction $=0.13$ ). Similar trends were reported when comparing HEC vs EC. The investigators went onto evaluate $61 \mathrm{HER} 2+$ tumors for TOP2A amplification by FISH, defining amplification as a gene copy:CEP17 ratio of $\geq 1.5$. For those with HER2/TOP2A co-amplification, EFS was better with anthracycline-containing arms (HEC or EC) vs CMF. Those without TOP2A amplification did not appear to benefit from anthracycline.

Samples from the MA-5 study were also evaluated for TOP $2 A^{150}$. While HER2 had been determined in 639 (90\%) of tumors from whole sections, TOP2A was able to be determined for only 438 $(62 \%)$ of samples. A clear trend toward differential RFS and OS benefit with anthracycline-based therapy in TOP2A amplified (ratio $>2$ ) cancers was noted but did not meet statistical significance. The hazard ratio for RFS for CEF vs CMF in those with TOP2A amplified tumors $(N=54)$ was $0.51(p=0.20)$ and was 0.90 for TOP2A normal $(N=358)$ tumors.

A larger analysis was performed on samples from the DBCG 89D trial in which patients were treated with adjuvant $C M F$ or $C^{8} F^{81,147}$. While there was no differential benefit from anthracycline-based therapy in patients with HER2 + vs HER2 - breast cancer in this study, those with tumor TOP2A-amplification $(N=92)($ ratio $\geq 2)$ had a significantly improved RFS and OS with CEF. Those with TOP2A-normal tumors $(N=589)$ derived no differential benefit with anthracycline-based therapy.

Two combined analyses of samples from the BR9601 and NEAT studies, in which patients were treated with adjuvant CMF or CMFepirubicin were also published ${ }^{84,85}$. There was no association between TOP2A-amplification and differential benefit from anthracycline. However, as previously pointed out, the cutoff for TOP $2 A$ amplification was set at a gene copy ratio of $>1.5$. rather than the more accepted $>2$. As a result, a number of TOP $2 A$ normal tumors were likely included in the TOP $2 A$ "amplified" group.

Two meta-analyses ${ }^{87,176}$ were conducted on the same five similarly designed studies comparing adjuvant CMF vs anthracycline-based chemotherapy (DBCG 89d, BR9601, NEAT, $M A-5$, and the Belgian trial) to address whether the TOP $2 A$ alteration predicts benefit from an anthracycline. The first ${ }^{87}$, published in 2011, is described in detail above and demonstrated that the improved outcome associated with anthracyclines appeared to be restricted to patients with HER2-amplified tumors. Of 3102 samples tested for TOP2A, 275 (9\%) were amplified (ratio $>2$ ). Those with TOP2A-amplification showed a greater benefit from anthracycline vs CMF chemotherapy (EFS HR $=0.62$, OS HR $=0.67$ ). In contrast, no differential benefit was observed for anthracycline-based therapy vs CMF in the 2511 patients with TOP2A-normal tumors (EFS HR $=0.88$, OS HR $=0.89$ ). As pointed out above, testing a portion of these samples at a central laboratory showed a relatively low (69\%) concordance in TOP2A results, calling into question the reliability of these results. The authors acknowledge this by calling for increased standardization of TOP2A FISH testing and advising against the routine use of TOP2A testing to select patients for anthracycline-based treatment.

\section{TOP2A deletion}

From a biological standpoint, it does not make intuitive sense that patients with tumor TOP2A deletion would be more sensitive to anthracyclines. Indeed, early studies indicated deletion of TOP $2 A$ is associated with diminished expression of the protein and resistance to anthracycline ${ }^{125,127}$. However other studies have indicated that deletion of TOP2A does not correlate with reduced expression of Topolla ${ }^{148,177}$. Once again, the close connection between proliferation and Topolla expression during cell division only complicates the matter. In addition, analysis of tumor samples from several trials ${ }^{81,87,147,150}$ have reported that deletion of TOP $2 A$ may be associated with increased sensitivity and better outcome with anthracycline vs non-anthracycline-based therapy, though this finding is not consistent ${ }^{85}$. In the DBCG $89 \mathrm{~d}$, TOP2A deletions were reported in $\sim 11 \%$ and trended toward benefit from anthracycline-based therapy. When combining patients with amplification and deletion ("TOP2A altered"), this interaction between treatment and marker became significant ${ }^{81,147}$. An 


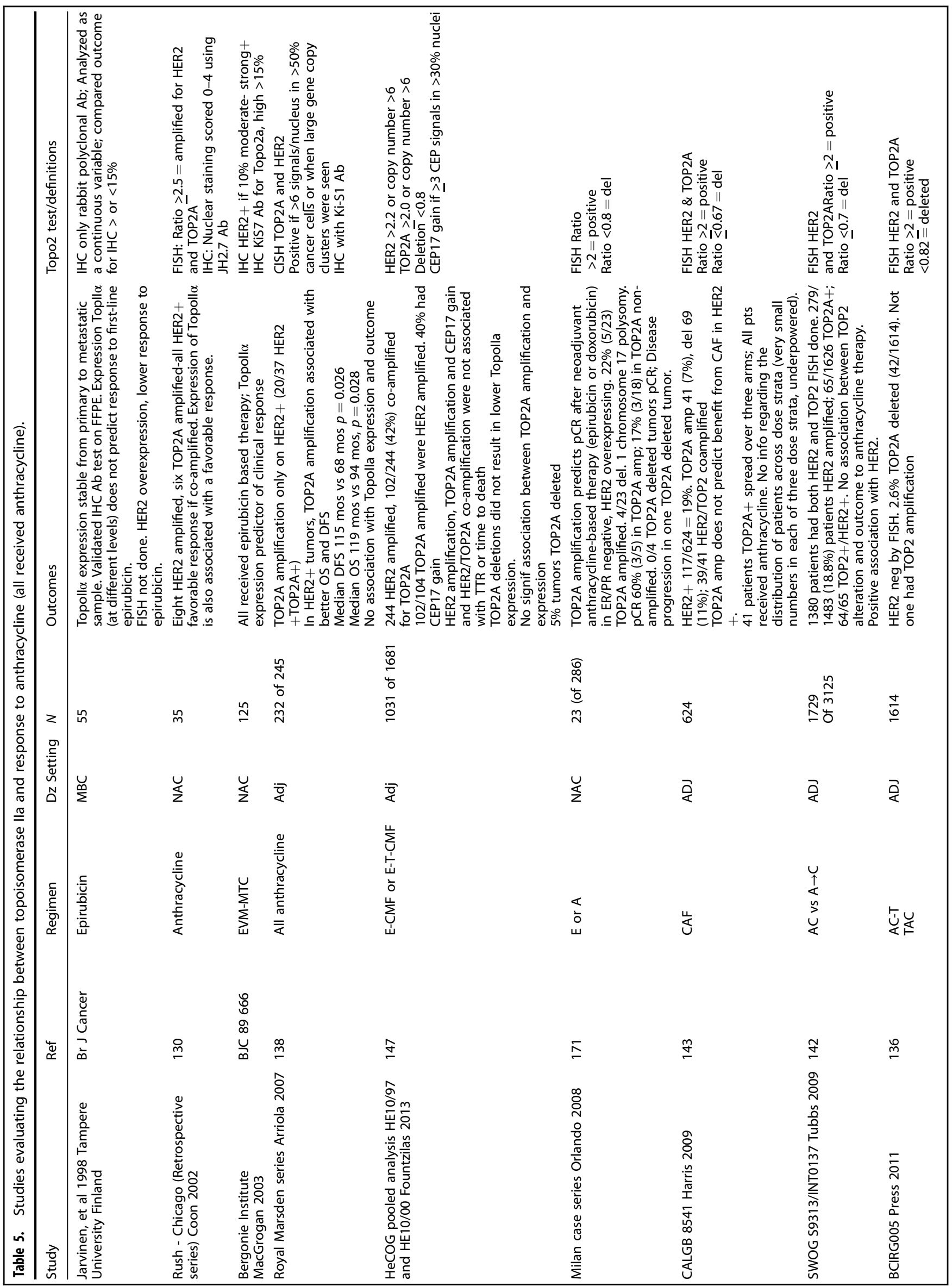




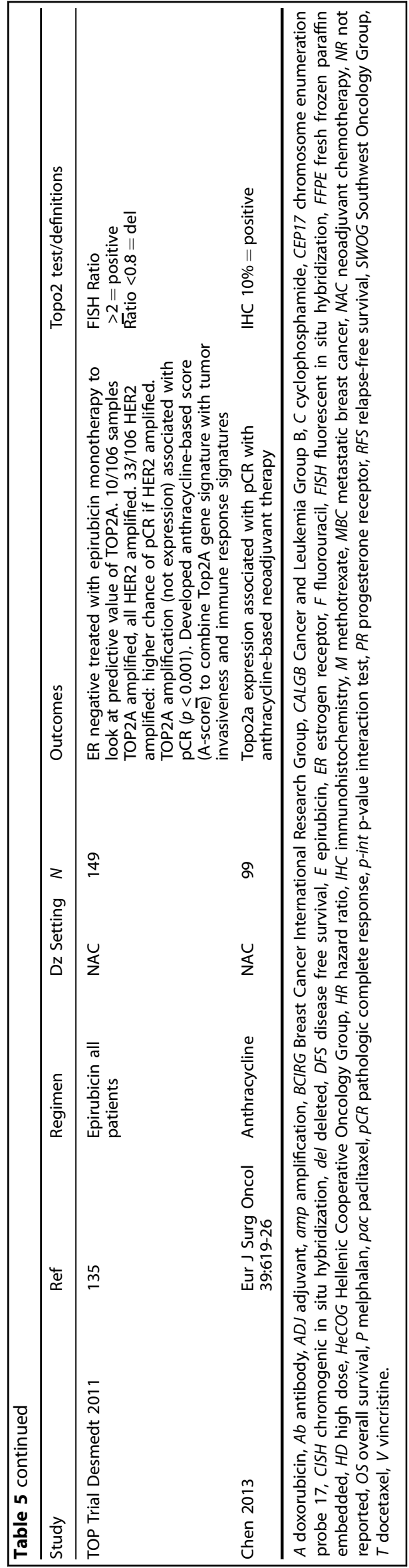

analysis of these samples using PAM-50 intrinsic subtyping published in $2020^{88}$ indicated that two-thirds of tumors designated as TOP2A deleted were actually HER2-enriched. Samples from the MA-5 trial ${ }^{150}$ were also tested and only 26 of 438 (6\%) were determined to be TOP $2 A$ deleted. Those with deletion appeared to gain substantial benefit from anthracyclines (RFS $\mathrm{HR}=0.16 p=0.02$, OS $\mathrm{HR}=0.18, p=0.07$ ), though the small sample size should be noted. When patients with either TOP2A alteration were combined, a significant benefit with CEF over CMF was observed (adjusted RFS HR $=0.35, p=0.005$; adjusted OS HR $=0.33, p=0.008$ ) whereas those with TOP2A normal tumors did similarly whether treated with CMF or CEF. The test for interaction between treatment and TOP2A status (altered vs not) trended toward significant RFS $(P=0.09)$ and was significant for OS $(P=$ 0.02). Both these studies defined TOP $2 A$ deletion as a ratio of $<0.8$. In contrast, the BR9601/NEAT analysis ${ }^{85}$ in which $11 \%(191 / 1 \overline{7} 62)$ patients had TOP2A deletion (ratio <0.8), demonstrated no significant interaction with benefit from anthracycline treatment and TOP2A del, TOP2A amplification, or TOP2A alteration. A metaanalysis including the above four studies plus the Belgian trial also looked at TOP2A deletions ${ }^{87}$. Of 3102 tumors, 316 (10\%) were TOP2A deleted (TOP2A/CEP17 ratio <0.8). Patients with TOP2A deletions seemed to benefit greater from anthracycline compared to those with normal tumors. Again, when all patients with TOP2A alterations were combined and compared to those with HER2 normal tumors, there was a significant interaction in favor of anthracyclines for TOP $2 A$ altered tumors (EFS $p$ interaction $=$ 0.0183 , OS $p$ interaction $=0.0455$ ). However, the lack of biological rationale to explain how TOP $2 A$ gene deletion might be associated with anthracycline benefit makes one wonder if there is a different explanation for this effect observed on retrospective studies, each with small patient numbers.

\section{The exploration of other genomic alterations}

As referenced above, Desmedt and colleagues analyzed tumor tissue from a neoadjuvant clinical trial of anthracycline-based therapy and showed that $\mathrm{pCR}$ was associated with TOP2A amplification ${ }^{136}$. As part of this study, they also developed an "A-score" comprised of a TOP2A gene signature and two signatures related to tumor invasion and immune response. They validated the A-score in two cohorts of patients treated with neoadjuvant anthracycline-based regimens. This signature was shown to be associated with a high negative predictive value for pCR in both HER+ and negative disease. It does not appear, however, to have been evaluated in a study with anthracycline- vs non-anthracycline-based therapy.

Investigators have also investigated duplication of chromosome 17 centromere (CEP17 dup) as a marker of sensitivity to anthracyclines. It should be noted that it is not rare to see increased numbers of CEP17 in breast cancer and, in particular, HER2 + breast cancer (Michael Press, MD, PhD, personal communication). It is not clear that these increased CEP17 numbers actually represent duplication. It is difficult to envision why increased copies of CEP17 would lead to anthracycline sensitivity. However, a number of groups analyzed breast tumor samples for CEP17 dup with varying definitions (e.g., $>1.86$ CEP17/cell or $>2.25$ CEP17/cell) with inconsistent results ${ }^{176,178,179}$.

It should be acknowledged that our understanding of the evolution of genomic alterations in cancer continues to advance. For example, whole genome sequencing, with its enriched view of genomic structure, has uncovered mechanisms, such as chromothripsis-or the rapid accumulation of hundreds of gene rearrangements over a very short period of time, that lead to silencing of some genes and the generation of "neo"chromosomes that may become focally replicated oncogenic drivers by a process now termed, "chromanasynthesis"180-183. These events may lead to a high degree of genomic complexity and drive the 


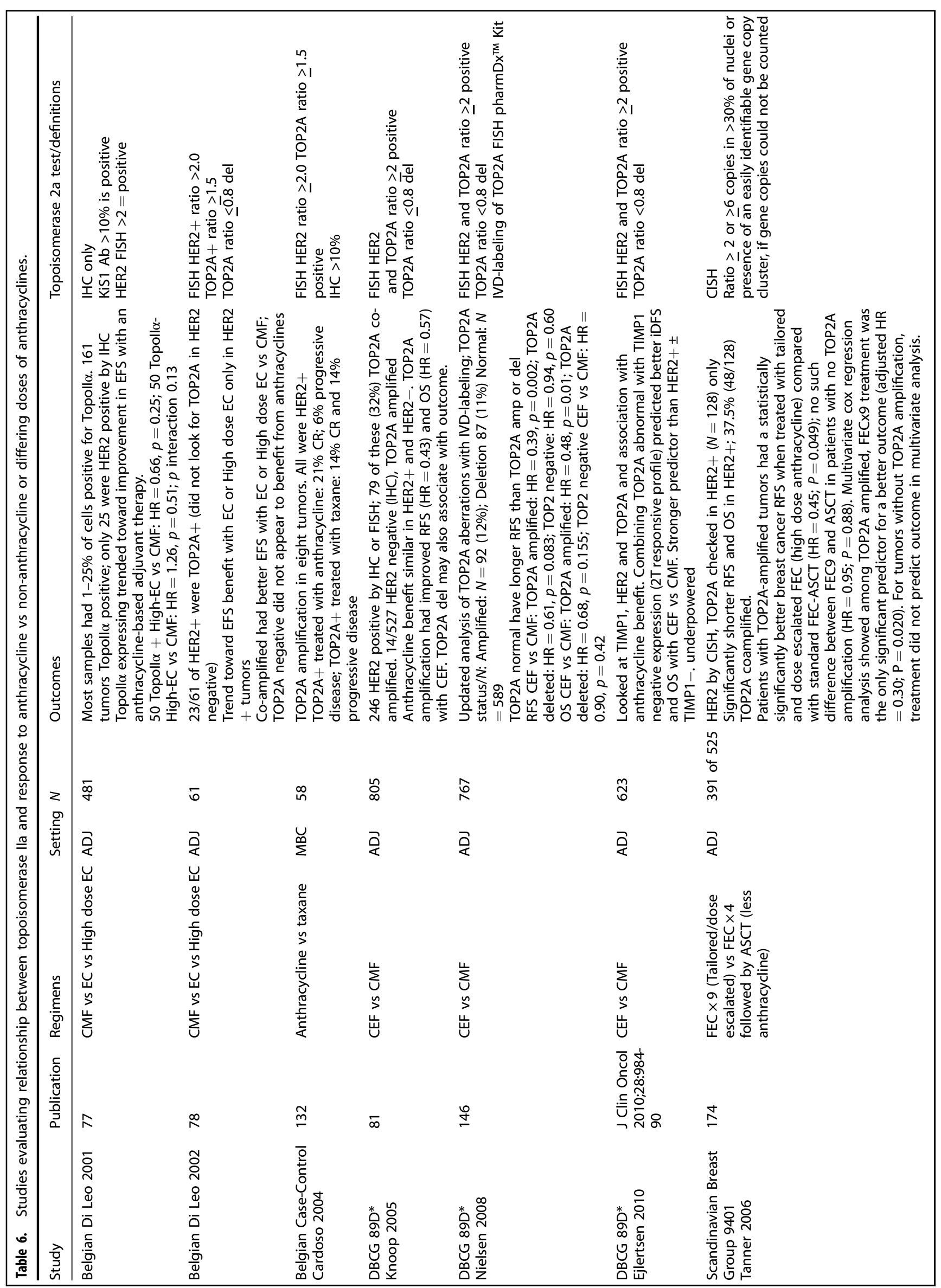




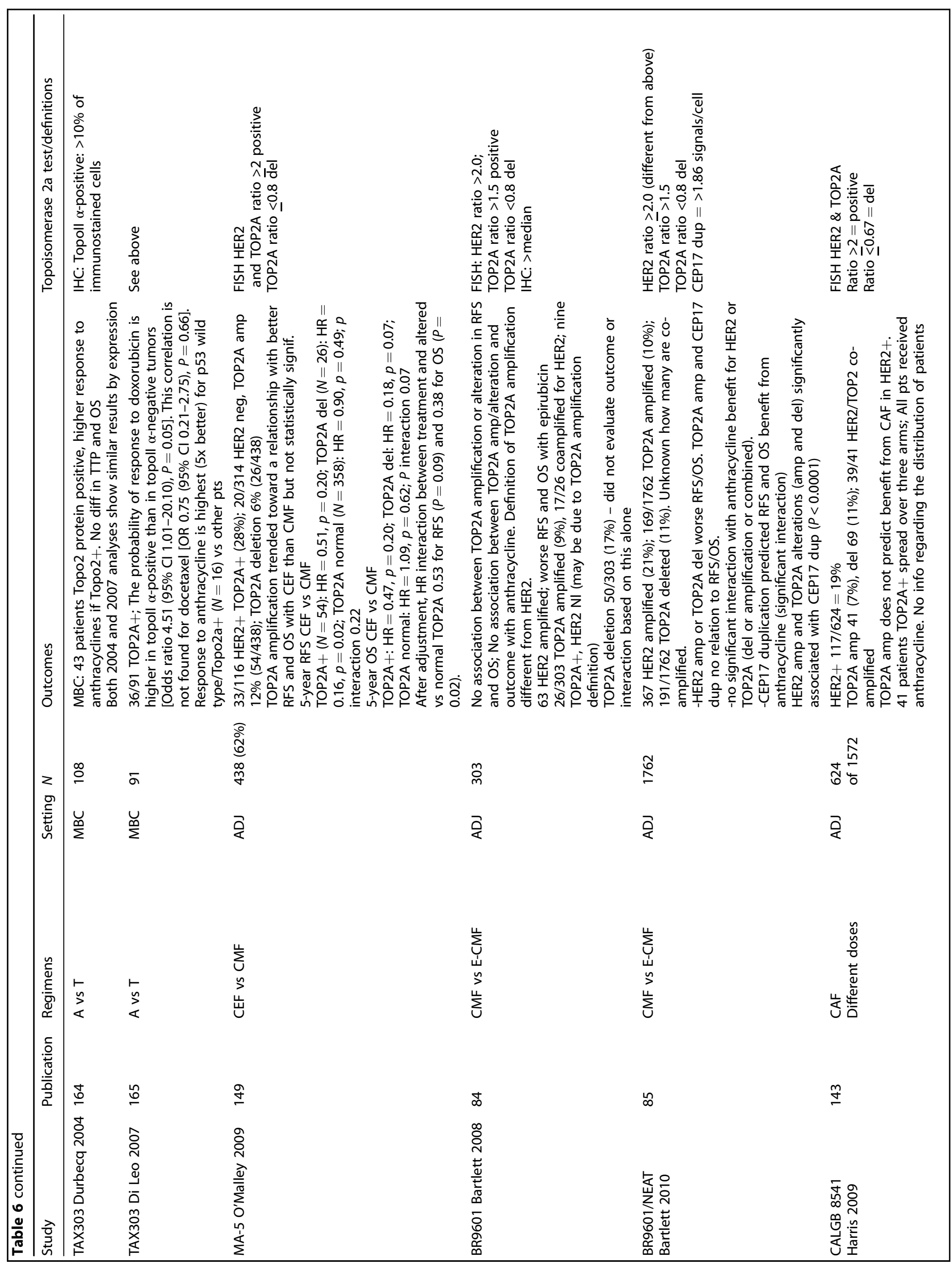




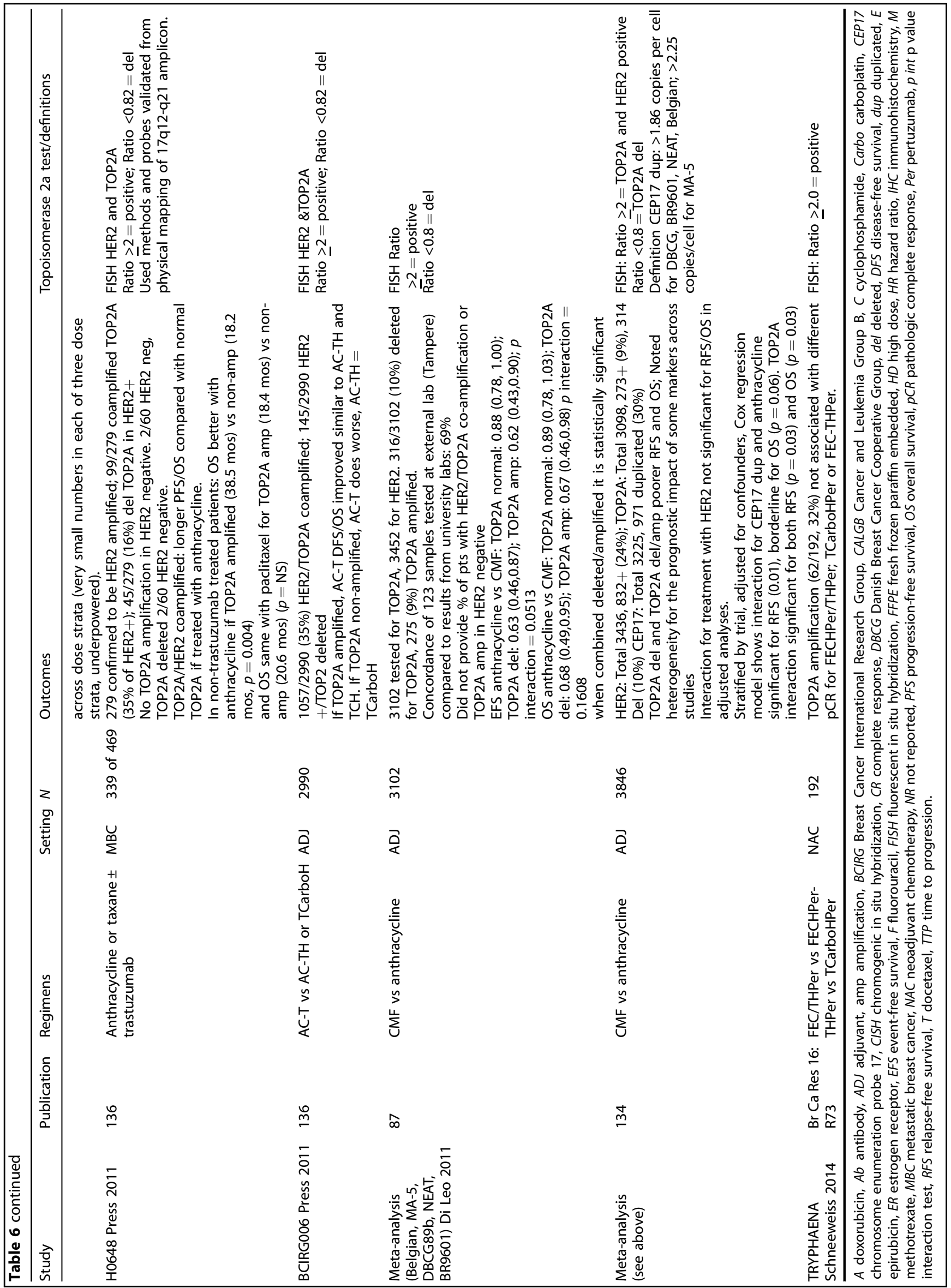


evolution of tumor response or resistance to therapy. Whether and how these types of alterations may impact the sensitivity of breast cancer to different types of chemotherapy has not yet been elucidated, though work in this area will hopefully shed further light.

\section{FIRST PROSPECTIVE EVALUATION OF ANTHRACYCLINE VS NON-ANTHRACYCLINE IN TOP2A NORMAL DISEASE}

All the above analyses relating to topoisomerase were performed retrospectively on tumor tissue from patients primarily treated with non-taxane-based chemotherapy. Tissue source, tumor quality, differing definitions of gene amplification, and interlaboratory discordance may all have significantly impacted results. Taken together, however, the majority of the evidence seemed seems to indicate that TOP2A alterations were associated with anthracycline benefit, but what was needed was a prospectively conducted randomized trial to evaluate whether anthracyclines benefit patients with TOP2A normal disease. The DBCG-07 READ trial was just this type of study ${ }^{184}$. Six cycles of docetaxel $(75 \mathrm{mg} /$ $\mathrm{m}^{2}$ ) and cyclophosphamide were compared to three cycles of EC followed by three cycles of docetaxel $\left(100 \mathrm{mg} / \mathrm{m}^{2}\right)$ in patients with early-stage, high risk, TOP $2 A$ normal breast cancer. In contrast to previous analyses ${ }^{87,176}$ TOP $2 A$ status was determined by an FDA approved FISH assay (TOP2A pharmDX; Dako A/S, Glostrup, Denmark) at one of three laboratories using a signal-tocentromere 17 ratio of $0.8-1.9$ as the definition of normal. A total of 2012 patients were recruited to the trial and were followed for a median of 69 months. In this prospective study, there was no significant difference between the two groups with regard to DFS $(\mathrm{HR}=1.00, p=1.00)$ distant DFS (HR=1.12, $p=.40)$ or $\mathrm{OS}(\mathrm{HR}=$ $1.15, p=0.41)$. No difference in outcome was noted based on estrogen receptor status or Ki67, both of which. were tested locally. Counterintuitively, subset analysis seemed to indicate those with lower-grade tumors and postmenopausal patients benefit more from EC-D. It is unclear if this finding is real and if so, whether this is due to a higher dose of docetaxel or the addition of the anthracycline. Regardless, these data do not support the claim that anthracyclines preferentially benefit those with TNBC or high-grade tumors.

One interesting point to note is that the Danish investigators carried out central TOP2A analysis in 5153 patients prior to enrolling to this study and identified 835 with a TOP2A alteration. Ongoing analyses are planned to evaluate what percentage of patients had TOP $2 A$ amplification vs deletion and how the alteration correlated with HER2 status (personal communication Bent Ejlertsen).

The BCIRG005 analysis of 1614 samples tested centrally for HER2 and TOP2A demonstrated zero cases of TOP $2 A$ amplification in $H E R 2$ normal tumors ${ }^{137}$. This calls into question whether false positives account for the reporting of TOP2A positive, HER2 normal tumors. Only $42(2.6 \%)$ of tumors were determined to be TOP2A deleted in the BCIRG analysis and it was not associated with differential DFS or OS in this study in which all patients received anthracyclines.

\section{THERE IS NO ROLE OF ANTHRACYCLINES IN HER2+ DISEASE REGARDLESS OF TOP2A AMPLIFICATION}

Though HER2 amplified tumors displayed varying sensitivity to anthracycline-based regimens described above, the introduction of trastuzumab revolutionized systemic therapy in this patient population. After improved TTP and OS was shown with the addition of trastuzumab to chemotherapy in metastatic HER2 amplified breast cancer ${ }^{185}$, further studies in early-stage HER2 amplified breast cancer ${ }^{186-188}$ demonstrated significant DFS and OS benefit with the addition of trastuzumab to standard chemotherapy. However, as all three large studies used a combination of trastuzumab and an anthracycline, there was also a four to fivefold increase in the rate of CHF.

A fourth study (BCIRG006) assigned patients with early-stage HER2 amplified breast cancer to receive either AC-T, AC-T +trastuzumab, or a new anthracycline-sparing, platinumcontaining regimen: $\mathrm{TCH}$ (docetaxel, carboplatin, and trastuzumab) ${ }^{189}$. This regimen was based on preclinical studies showing synergy with platinum salts and trastuzumab, which was not evident with anthracyclines or taxanes ${ }^{190-192}$. As expected, each trastuzumab-containing group had improved DFS and OS compared to the AC-T arm. Though the statistical plan was to compare each trastuzumab arm head-to-head with the control arm, a post hoc statistical comparison between the two trastuzumab arms was performed, revealing no difference in efficacy with respect to DFS or OS.

Anthracycline-free regimens have also been evaluated in the neoadjuvant setting for HER2 + breast cancer. In 2020, results from neoCART, a phase II neoadjuvant study comparing TCH to EC-TH were presented ${ }^{122}$. Of 131 treated patients, two-thirds had node involvement. The tpCR rate was $56 \%$ with $\mathrm{TCH}$ and $38.5 \%$ with $\mathrm{EC}-\mathrm{TH}$ $(p=0.044)$. Furthermore, two studies (TRYPHAENA and TRAIN-2) examined neoadjuvant trastuzumab and pertuzumab-containing regimens for HER2 amplified, early-stage breast cancer and showed no significant difference in the rates of pCR or EFS with or without an anthracycline ${ }^{193-195}$. It is notable that in TRAIN-2, patients in the nonanthracycline arm received nine cycles of weekly paclitaxel plus carboplatin with trastuzumab plus pertuzumab. In the anthracycline arm, a total of five chemotherapy agents plus pertuzumab/ trastuzumab (5'fluorouracil, epirubicin, cyclophosphamide, carboplatin and paclitaxel, FEC-HP $\rightarrow$ TCHP) were given. In spite of the fact that the anthracycline arm received more chemotherapy agents, the $\mathrm{pCR}$ rate and EFS rates were identical. Some argue that anthracyclinebased therapy should be reserved for those with the highest risk of relapse. In contrast, however, subset analyses of BCIRG006 and TRAIN-2 demonstrate that the recurrences for patients with four or more LN involved was not improved by the addition of an anthracycline to trastuzumab-based therapy ${ }^{195,196}$. In fact, the TRAIN-2 study showed a trend toward a better outcome with the non-anthracycline treatment in this high-risk group.

In terms of safety, treatment-related leukemic events were noted in the anthracycline arms of both TRAIN-2 and BCIRG006. Moreover, significantly higher rates of cardiac toxicity were observed in the anthracycline arms of these studies, with sustained cardiac dysfunction noted during follow up.

It is noteworthy that in BCIRG006, all patients had centrally confirmed HER2 amplified tumors. Central testing for TOP2A was also performed on samples from 2990 patients and demonstrated that 35\% (1057) had TOP2A co-amplification and 145 (5\%) had TOP2A deletion ${ }^{137}$. For TOP2A normal tumors, TCH and AC-TH were similarly associated with a significantly improved DFS and OS compared to AC-T. Those with TOP2A amplification benefited equally from $\mathrm{AC}-\mathrm{T}, \mathrm{AC}-\mathrm{TH}$, and $\mathrm{TCH}$, suggesting that in HER2amplified tumors with TOP $2 A$ co-amplification, a similar outcome is achieved with targeting one vs both alterations. Thus, inhibiting both TOP2A (with doxorubicin) and HER2 (with trastuzumab) in the AC-TH arm does not appear to improve efficacy. Some would argue these patients could thus avoid trastuzumab and just use AC-T, while others would conclude that the safer $\mathrm{TCH}$ regimen should be used to maximize the therapeutic index.

These trials provide the best evidence that, with HER2-directed therapy, there is no significant benefit of adding anthracyclines to neo/adjuvant regimens in early-stage breast cancer. Accordingly, in 2021, the National Comprehensive Cancer Network Guidelines ${ }^{197}$ removed anthracycline-based therapy from the list of "preferred regimens" for the treatment of HER2+ early-stage breast cancer and into the category of regimens for use in certain situations. One notable situation where an anthracycline would be appropriate to consider is for a pregnant woman 
diagnosed with a HER2 + breast cancer. In this situation, the use of trastuzumab is contraindicated and the standard approach would be to proceed with anthracycline-based chemotherapy.

\section{WHY DO WE CARE? TOXICITY OF ANTHRACYCLINES}

One of the most recognized consequences of anthracycline use is myocardial injury. Although heart failure is widely acknowledged to be associated with these drugs, measuring the true incidence of heart damage remains elusive, due to limited long-term studies in asymptomatic patients, the presence of confounders including cardiac risk factors and concomitant use of other cardiotoxic cancer therapies and the retrospective nature of many studies aimed at gauging rates of cardiac dysfunction. The large adjuvant trastuzumab studies provide unique insights into cardiac outcomes in those treated with anthracyclines or anthracyclines followed by trastuzumab. For example, the NCCTG N9831 and NSABP B31 trials reported that $0.6-1.3 \%$ of patients treated with AC-T and $3.0-4.0 \%$ of patients treated with AC-TH developed a cardiac event during the 6- to 7-year follow up, respectively. Though these rates seem somewhat low, it is important to call out that $5-7 \%$ of patients developed a cardiac event during four cycles of AC chemotherapy that precluded them from proceeding to trastuzumab (or placebo)-based therapy. These studies, in which serial left ventricular ejection fraction (LVEF) measurement was required prior to and after treatment with four cycles of $A C$ revealed higher rates of heart damage than was perhaps previously recognized since patients needed to meet certain heart function criteria, even if they had no symptoms of heart damage, in order to proceed onto trastuzumab-based therapy ${ }^{198,199}$. That said, these studies only measured LVEF during or shortly after active treatment (i.e., 18-21 months), thus long-term subclinical cardiac damage is likely underreported. In contrast, BCIRG006, followed all patients with cardiac function measurements long-term. At 10 years, $1.96 \%$ of patients assigned to AC-TH, $0.76 \%$ of those assigned to AC-T, and $0.37 \%$ of those assigned to $\mathrm{TCH}$ developed $\mathrm{CHF}^{196}$. Importantly, LVEF decline $>10 \%$ from baseline was noted in 19,12, and $9 \%$ of those treated with AC-TH, $A C-T$, and $T C H$, respectively. While the mean drop in LVEF was transient in $\mathrm{TCH}$-treated patients, it did not recover to baseline in those treated with an anthracycline. Keeping in mind clinical trial patients tended to be younger with a low incidence of cardiac risk factors due to screening requirements, it is likely these cardiac outcomes would be worse in a real-world setting.

In addition to the more common cardiac effects of anthracyclines, another life-threatening complication is therapy related myelodysplastic syndrome and acute myeloid leukemia (t-MDS/ $A M L$ ) resulting from acquired somatic mutations in hematopoietic stem/progenitor cells. Topoisomerase II inhibitors contribute to leukemogenesis by inducing chromosomal breakages and translocations, in regions known to cause malignant transformation, such as $11 \mathrm{q} 23,21 \mathrm{q} 22$, inv(16), $\mathrm{t}(15,17)$, and $\mathrm{t}(9,22)^{200,201}$. Large studies suggest that the overall incidence of anthracyclineassociated $\mathrm{t}-\mathrm{MDS} / \mathrm{AML}$, while greater than that of the general population, remains $<1 \%$, with 10 -year cumulative risks ranging from $0.2-1.7 \% 202$. This risk seems to increase with a greater cumulative dose of anthracyclines, especially when combined with cyclophosphamide. Of note, standard dose cyclophosphamide in the absence of an anthracycline does not seem to increase the risk of MDS/AML compared to the general population ${ }^{202}$.

It should, of course, be acknowledged that non-anthracycline regimens can also have distressing toxicity, such as neuropathy in the case of taxane/platinum regimens, and permanent alopecia which occurs in a dose-dependent fashion in a minority of patients with docetaxel, though these toxicities are not lifethreatening.

While the absolute risk of anthracycline-related life-threatening toxicities $(<5 \%$ for CHF, leukemia, and MDS) may seem inconsequential in the fight to avoid a metastatic recurrence of breast cancer, it is sobering to consider that for most patients, the absolute benefit expected to be imparted by adding an anthracycline to taxane-based therapy is similarly less than $5 \%$.

\section{CONCLUSION}

The following statement is true: as of this writing: there has been no prospective randomized trial that has demonstrated an OS benefit from the addition of anthracyclines to taxane-based chemotherapy in the curative setting. Although HER2 amplification was thought to indicate a tumor subtype that would benefit from the addition of an anthracycline in an era that predated trastuzumab, no randomized study has shown the addition of anthracycline to a taxane/trastuzumab-based regimen improves outcomes for HER2-amplified breast cancer. While multiple markers have been postulated from retrospective analyses to identify those who will benefit from an anthracycline, most results were inconsistent and only one -TOP2A- has been evaluated prospectively. This marker may be the most biologically sound one tested as it is the target of the anthracyclines. Only one study has prospectively tested whether an anthracycline-based regimen adds benefit to TOP2A normal tumors and demonstrated no DFS or OS improvement ${ }^{184}$. In biomarker unselected patients with HER2disease, a small absolute DFS benefit with the addition of an anthracycline to taxane-based therapy was noted in one study ${ }^{109}$, though several other trials did not confirm this benefit ${ }^{62,110,111}$. It is true that subset analyses of a handful of trials do suggest that for those patients with the heaviest disease burden (e.g., four or more nodes involved), the addition of an anthracycline to multiagent chemo regimens improves DFS ${ }^{109,111}$. However, in the era of mass breast cancer screening, fortunately, the majority of patients are not diagnosed with such locally advanced cancer. As we move into a future where we will likely be incorporating more biologically targeted therapies for those with the high-risk disease-a PARP inhibitor, olaparib, was just approved in the adjuvant setting for BRCA-mutation carriers and an adjuvant CDK4/6 inhibitor may indeed be available by the time this paper is published-the potential incremental benefits of adding an anthracycline will likely diminish further. Thus, as we select patients whose disease burden warrants the incorporation of an anthracycline into their regimen, we must also consider carefully that the potential life-altering toxicities associated with anthracyclines are real and are likely underreported. Thus, rather than asking which patients can be safely be treated without an anthracycline, we should be asking, does the data clearly exist to warrant the use of an anthracycline, keeping in mind that in many cases we are potentially harming patients more than helping them.

\section{DATA AVAILABILITY}

Authors can confirm that all relevant data are included in the paper and/or its supplementary information files.

Received: 20 July 2021; Accepted: 21 September 2021; Published online: 08 October 2021

\section{REFERENCES}

1. Fisher, B. et al. Surgical adjuvant chemotherapy in cancer of the breast: results of a decade of cooperative investigation. Ann. Surg. 168, 337-356 (1968).

2. Noer, R. J. Adjuvant chemotherapy. Thio-tepa with radical mastectomy in the treatment of breast cancer. Am. J. Surg. 106, 405-412 (1963).

3. Peto, R. et al. Comparisons between different polychemotherapy regimens for early breast cancer: meta-analyses of long-term outcome among 100,000 women in 123 randomised trials. Lancet 379, 432-444 (2012).

4. Siegel, R. L., Miller, K. D. \& Jemal, A. Cancer statistics, 2020. CA Cancer J. Clin. 70, 7-30 (2020). 
5. Fisher, B., Slack, N., Katrych, D. \& Wolmark, N. Ten year follow-up results of patients with carcinoma of the breast in a co-operative clinical trial evaluating surgical adjuvant chemotherapy. Surg. Gynecol. Obstet. 140, 528-534 (1975).

6. Fisher, B., Jeong, J. H., Anderson, S. \& Wolmark, N. Treatment of axillary lymph node-negative, estrogen receptor-negative breast cancer: updated findings from National Surgical Adjuvant Breast and Bowel Project clinical trials. J. Nat Cancer Inst. 96, 1823-1831 (2004).

7. Fisher, B., Fisher, E. R. \& Redmond, C. Ten-year results from the National Surgical Adjuvant Breast and Bowel Project (NSABP) clinical trial evaluating the use of L-phenylalanine mustard (L-PAM) in the management of primary breast cancer. J. Clin. Oncol. 4, 929-941 (1986).

8. Fisher, B. et al. Treatment of primary breast cancer with chemotherapy and tamoxifen. N. Engl. J. Med. 305, 1-6 (1981).

9. Hutchins, L. F. et al. Randomized, controlled trial of cyclophosphamide, methotrexate, and fluorouracil versus cyclophosphamide, doxorubicin, and fluorouracil with and without tamoxifen for high-risk, node-negative breast cancer: treatment results of Intergroup Protocol INT-0102. J. Clin. Oncol. 23, 8313-8321 (2005).

10. Tamoxifen for early breast cancer: an overview of the randomised trials. Early Breast Cancer Trialists' Collaborative Group. Lancet 351, 1451-1467 (1998).

11. Jonat, W. et al. Goserelin versus cyclophosphamide, methotrexate, and fluorouracil as adjuvant therapy in premenopausal patients with node-positive breast cancer: the Zoladex Early Breast Cancer Research Association Study. J. Clin. Oncol. 20, 4628-4635 (2002).

12. Hackshaw, A. et al. Long-term effectiveness of adjuvant goserelin in premenopausal women with early breast cancer. J. Natl Cancer Inst. 101, 341-349 (2009).

13. Fisher, B. et al. Influence of tumor estrogen and progesterone receptor levels on the response to tamoxifen and chemotherapy in primary breast cancer. J. Clin. Oncol. 1, 227-241 (1983).

14. Nissen-Meyer, R., Kjellgren, K., Malmio, K., Mansson, B. \& Norin, T. Surgical adjuvant chemotherapy: results with one short course with cyclophosphamide after mastectomy for breast cancer. Cancer 41, 2088-2098 (1978).

15. Bonadonna, G. et al. 30 years' follow up of randomised studies of adjuvant CMF in operable breast cancer: cohort study. Bmj 330, 217 (2005).

16. Blum, R. H. \& Carter, S. K. Adriamycin. A new anticancer drug with significant clinical activity. Ann. Intern. Med. 80, 249-259 (1974).

17. Jones, S. E., Durie, B. G. \& Salmon, S. E. Combination chemotherapy with adriamycin and cyclophosphamide for advanced breast cancer. Cancer 36, 90-97 (1975).

18. Corbett, T. H., Griswold, D. P., Mayo, J. G., Laster, W. R. \& Schabel, F. M. Jr Cyclophosphamide-adriamycin combination chemotherapy of transplantable murine tumors. Cancer Res. 35, 1568-1573 (1975).

19. Smalley, R. V., Carpenter, J., Bartolucci, A., Vogel, C. \& Krauss, S. A comparison of cyclophosphamide, adriamycin, 5-fluorouracil (CAF) and cyclophosphamide, methotrexate, 5-fluorouracil, vincristine, prednisone (CMFVP) in patients with metastatic breast cancer: a Southeastern Cancer Study Group Project. Cancer 40, 625-632 (1977)

20. Bull, J. M. et al. A randomized comparative trial of adriamycin versus methotrexate in combination drug therapy. Cancer 41, 1649-1657 (1978).

21. Buzdar, A. U. et al. Postoperative adjuvant chemotherapy with fluorouracil, doxorubicin, cyclophosphamide, and BCG vaccine. A follow-up report. JAMA 242, 1509-1513 (1979).

22. Casper, E. S. et al. Combined modality treatment of locally advanced breast cancer: adjuvant combination chemotherapy with and without doxorubicin. Breast Cancer Res. Treat. 9, 39-44 (1987).

23. Fisher, B. et al. Doxorubicin-containing regimens for the treatment of stage II breast cancer: the National Surgical Adjuvant Breast and Bowel Project experience. J. Clin. Oncol. 7, 572-582 (1989).

24. Fisher, B. et al. Two months of doxorubicin-cyclophosphamide with and without interval reinduction therapy compared with 6 months of cyclophosphamide, methotrexate, and fluorouracil in positive-node breast cancer patients with tamoxifen-nonresponsive tumors: results from the National Surgical Adjuvant Breast and Bowel Project B-15. J. Clin. Oncol. 8, 1483-1496 (1990).

25. Moliterni, A., Bonadonna, G., Valagussa, P., Ferrari, L. \& Zambetti, M. Cyclophosphamide, methotrexate, and fluorouracil with and without doxorubicin in the adjuvant treatment of resectable breast cancer with one to three positive axillary nodes. J. Clin. Oncol. 9, 1124-1130 (1991).

26. De Placido, S. et al. CMF vs alternating CMF/EV in the adjuvant treatment of operable breast cancer. A single centre randomised clinical trial (Naples GUN-3 study). Br. J. Cancer 71, 1283-1287 (1995).

27. Bang, S. M. et al. Adjuvant doxorubicin and cyclophosphamide versus cyclophosphamide, methotrexate, and 5-fluorouracil chemotherapy in premenopausal women with axillary lymph node positive breast carcinoma. Cancer 89, 2521-2526 (2000).
28. Mauriac, L., Durand, M., Chauvergne, J., Dilhuydy, J. M. \& Bonichon, F. Randomized trial of adjuvant chemotherapy for operable breast cancer comparing i.v. CMF to an epirubicin-containing regimen [see comment]. Ann. Oncol. 3, 439-443 (1992)

29. Budd, G. T. et al. Short-course FAC-M versus 1 year of CMFVP in node-positive, hormone receptor-negative breast cancer: an intergroup study. J. Clin. Oncol. 13, 831-839 (1995).

30. Carpenter, J. T., Velez-Garcia, E. \& Aron, B. S. et al. Five-year results of a randomized comparison of cyclophosphamide, doxorubicin (adriamycin) and fluorouracil (CAF) vs cyclophosphamide, methotrexate and fluorouracil (CMF) in node positive breast cancer: a Southeastern Cancer Study Group study. Proc. Am. Soc. Clin. Oncol. 13, 66 (1994)

31. Levine, M. N. et al. A randomized trial comparing 12 weeks versus 36 weeks of adjuvant chemotherapy in stage II breast cancer. J. Clin. Oncol. 8, 1217-1225 (1990).

32. Coombes, R. C. et al. Adjuvant cyclophosphamide, methotrexate, and fluorouracil versus fluorouracil, epirubicin, and cyclophosphamide chemotherapy in premenopausal women with axillary node-positive operable breast cancer: results of a randomized trial. The International Collaborative Cancer Group. J. Clin. Oncol.14, 35-45 (1996)

33. Piccart, M. J. et al. Phase III trial comparing two dose levels of epirubicin combined with cyclophosphamide with cyclophosphamide, methotrexate, and fluorouracil in node-positive breast cancer. J. Clin. Oncol. 19, 3103-3110 (2001).

34. Fisher, B. et al. Tamoxifen and chemotherapy for axillary node-negative, estrogen receptor-negative breast cancer: findings from National Surgical Adjuvant Breast and Bowel Project B-23. J. Clin. Oncol. 19, 931-942 (2001).

35. Levine, M. N. et al. Randomized trial of intensive cyclophosphamide, epirubicin, and fluorouracil chemotherapy compared with cyclophosphamide, methotrexate, and fluorouracil in premenopausal women with node-positive breast cancer. National Cancer Institute of Canada Clinical Trials Group. J. Clin. Oncol. 16 2651-2658 (1998).

36. De Placido, S. et al. A randomised factorial trial of sequential doxorubicin and CMF vs CMF and chemotherapy alone vs chemotherapy followed by goserelin plus tamoxifen as adjuvant treatment of node-positive breast cancer. Br. J. Cancer 92, 467-474 (2005).

37. Misset, J. L. et al. Adjuvant treatment of node-positive breast cancer with cyclophosphamide, doxorubicin, fluorouracil, and vincristine versus cyclophosphamide, methotrexate, and fluorouracil: final report after a 16-year median follow-up duration. J. Clin. Oncol. 14, 1136-1145 (1996).

38. Ejlertsen, B. et al. Improved outcome from substituting methotrexate with epirubicin: results from a randomised comparison of CMF versus CEF in patients with primary breast cancer. Eur. J. Cancer 43, 877-884 (2007).

39. Poole, C. J. et al. Epirubicin and cyclophosphamide, methotrexate, and fluorouracil as adjuvant therapy for early breast cancer. N. Engl. J. Med. 355, 1851-1862 (2006)

40. Martin, M. et al. Doxorubicin in combination with fluorouracil and cyclophosphamide (i.v. FAC regimen, day 1,21) versus methotrexate in combination with fluorouracil and cyclophosphamide (i.v. CMF regimen, day 1,21) as adjuvant chemotherapy for operable breast cancer: a study by the GEICAM group. Ann. Oncol. 14, 833-842 (2003).

41. Early Breast Cancer Trialists' Collaborative Group. Effects of chemotherapy and hormonal therapy for early breast cancer on recurrence and 15-year survival: an overview of the randomised trials. Lancet 365, 1687-1717 (2005).

42. Wani, M. C., Taylor, H. L., Wall, M. E., Coggon, P. \& McPhail, A. T. Plant antitumor agents. VI. The isolation and structure of taxol, a novel antileukemic and antitumor agent from Taxus brevifolia. J. Am. Chem. Soc. 93, 2325-2327 (1971).

43. Lavelle, F., Gueritte-Voegelein, F. \& Guenard, D. Taxotere: from yew's needles to clinical practice. Bull. Cancer 80, 326-338 (1993).

44. Gligorov, J. \& Lotz, J. P. Preclinical pharmacology of the taxanes: implications of the differences. Oncologist 9, 3-8 (2004).

45. Chan, S. et al. Prospective randomized trial of docetaxel versus doxorubicin in patients with metastatic breast cancer. J. Clin. Oncol. 17, 2341-2354 (1999).

46. Martin, M. et al. Adjuvant docetaxel for node-positive breast cancer. N. Engl. J. Med. 352, 2302-2313 (2005)

47. Roche, $H$. et al. Sequential adjuvant epirubicin-based and docetaxel chemotherapy for node-positive breast cancer patients: the FNCLCC PACS 01 Trial. J. Clin. Oncol. 24, 5664-5671 (2006).

48. Henderson, I. C. et al. Improved outcomes from adding sequential Paclitaxel but not from escalating Doxorubicin dose in an adjuvant chemotherapy regimen for patients with node-positive primary breast cancer. J. Clin. Oncol. 21, 976-983 (2003).

49. Mamounas, E. P. et al. Paclitaxel after doxorubicin plus cyclophosphamide as adjuvant chemotherapy for node-positive breast cancer: results from NSABP B-28. J. Clin. Oncol. 23, 3686-3696 (2005). 
50. Martin, M. et al. Randomized phase 3 trial of fluorouracil, epirubicin, and cyclophosphamide alone or followed by Paclitaxel for early breast cancer. J. Natl Cancer Inst. 100, 805-814 (2008).

51. Gianni, L. et al. Phase III trial evaluating the addition of paclitaxel to doxorubicin followed by cyclophosphamide, methotrexate, and fluorouracil, as adjuvant or primary systemic therapy: European cooperative trial in operable breast cancer. J. Clin. Oncol. 27, 2474-2481 (2009).

52. Francis, P. et al. Adjuvant chemotherapy with sequential or concurrent anthracycline and docetaxel: Breast International Group 02-98 randomized trial. J. Natl Cancer Inst. 100, 121-133 (2008).

53. Evans, T. R. et al. Phase III randomized trial of doxorubicin and docetaxel versus doxorubicin and cyclophosphamide as primary medical therapy in women with breast cancer: an anglo-celtic cooperative oncology group study. J. Clin. Oncol. 23, 2988-2995 (2005).

54. Gines, J. et al. Efficacy of taxanes as adjuvant treatment of breast cancer: a review and meta-analysis of randomised clinical trials. Clin. Transl. Oncol. 13, 485-498 (2011).

55. De Laurentiis, M. et al. Taxane-based combinations as adjuvant chemotherapy of early breast cancer: a meta-analysis of randomized trials. J. Clin. Oncol. 26, 44-53 (2008)

56. Legha, S. S., Tenney, D. M. \& Krakoff, I. R. Phase I study of taxol using a 5-day intermittent schedule. J. Clin. Oncol. 4, 762-766 (1986).

57. Jones, S. E. et al. Phase III trial comparing doxorubicin plus cyclophosphamide with docetaxel plus cyclophosphamide as adjuvant therapy for operable breast cancer. J. Clin. Oncol. 24, 5381-5387 (2006).

58. Valero, V. Docetaxel and cyclophosphamide in patients with advanced solid tumors. Oncology 11, 21-23 (1997).

59. Jones, S. et al. Docetaxel with cyclophosphamide is associated with an overall survival benefit compared with doxorubicin and cyclophosphamide: 7-year follow-up of US Oncology Research Trial 9735. J. Clin. Oncol. 27, 1177-1183 (2009).

60. Shulman, L. N. et al. Comparison of doxorubicin and cyclophosphamide versus single-agent paclitaxel as adjuvant therapy for breast cancer in women with 0 to 3 positive axillary nodes: CALGB 40101 (Alliance). J. Clin. Oncol. 32, 2311-2317 (2014).

61. Lee, K. S. et al. A randomized phase-III trial of docetaxel/capecitabine versus doxorubicin/cyclophosphamide as primary chemotherapy for patients with stage II/III breast cancer. Breast Cancer Res. Treat. 109, 481-489 (2008).

62. Delaloge, S. et al. Standard anthracycline based versus docetaxel-capecitabine in early high clinical and/or genomic risk breast cancer in the EORTC 10041/BIG 3-04 MINDACT phase III trial. J. Clin. Oncol. 38, 1186-1197 (2020).

63. Katsumata, N. et al. Phase III trial of doxorubicin plus cyclophosphamide (AC), docetaxel, and alternating $A C$ and docetaxel as front-line chemotherapy for metastatic breast cancer: Japan Clinical Oncology Group trial (JCOG9802). Ann. Oncol. 20, 1210-1215 (2009).

64. Watanabe, T. et al. Comparison of an AC-taxane versus AC-free regimen and paclitaxel versus docetaxel in patients with lymph node-positive breast cancer: final results of the National Surgical Adjuvant Study of Breast Cancer 02 trial, a randomized comparative phase 3 study. Cancer 123, 759-768 (2017).

65. Slamon, D. J. et al. Studies of the HER-2/neu proto-oncogene in human breast and ovarian. Cancer Sci. 244, 707-712 (1989).

66. Tandon, A. K., Clark, G. M., Chamness, G. C., Ullrich, A. \& McGuire, W. L. HER-2/neu oncogene protein and prognosis in breast. Cancer J. Clin. Oncol. 7, 1120-1128 (1989).

67. O'Reilly, S. M. et al. The relationship between c-erbB-2 expression, S-phase fraction and prognosis in breast cancer. Br. J. Cancer 63, 444-446 (1991).

68. Paik, S. et al. Pathologic findings from the National Surgical Adjuvant Breast and Bowel Project: prognostic significance of erbB-2 protein overexpression in primary breast cancer. J. Clin. Oncol. 8, 103-112 (1990).

69. Toikkanen, S., Helin, H., Isola, J. \& Joensuu, H. Prognostic significance of HER-2 oncoprotein expression in breast cancer: a 30-year follow-up. J. Clin. Oncol. 10, 1044-1048 (1992).

70. Gusterson, B. A. et al. Prognostic importance of c-erbB-2 expression in breast cancer. International (Ludwig) Breast Cancer Study Group. J. Clin. Oncol. 10, 1049-1056 (1992).

71. Allred, D. C. et al. HER-2/neu in node-negative breast cancer: prognostic significance of overexpression influenced by the presence of in situ carcinoma. J. Clin. Oncol. 10, 599-605 (1992).

72. Miles, D. W., Harris, W. H., Gillett, C. E., Smith, P. \& Barnes, D. M. Effect of c-erbB(2) and estrogen receptor status on survival of women with primary breast cancer treated with adjuvant cyclophosphamide/methotrexate/fluorouracil. Int. J. Cancer 84, 354-359 (1999).

73. Paik, S. et al. erbB-2 and response to doxorubicin in patients with axillary lymph node-positive, hormone receptor-negative breast cancer. J. Nat/ Cancer Inst. 90 , 1361-1370 (1998).
74. Paik, S. et al. HER2 and choice of adjuvant chemotherapy for invasive breast cancer: National Surgical Adjuvant Breast and Bowel Project Protocol B-15. J. Natl Cancer Inst. 92, 1991-1998 (2000).

75. Petruzelka, L. et al. C-erbB2 overexpression and treatment outcome in a randomized trial comparing adjuvant $\mathrm{CMF}$ and $\mathrm{AC}$ in equitoxic regiment in breast cancer. Proc. Am. Soc. Clin. Oncol. 19, Abstract 534 (2000).

76. De Laurentiis, M. C. F. et al. HER2 expression and anthracycline effect: result from the Naples GUN 3 randomized trial. J. Clin. Oncol. 20, 34 (2001).

77. Di Leo, A. et al. HER-2 and topo-isomerase llalpha as predictive markers in a population of node-positive breast cancer patients randomly treated with adjuvant CMF or epirubicin plus cyclophosphamide. Ann. Oncol. 12, 1081-1089 (2001).

78. Di Leo, A. et al. HER-2 amplification and topoisomerase llalpha gene aberrations as predictive markers in node-positive breast cancer patients randomly treated either with an anthracycline-based therapy or with cyclophosphamide, methotrexate, and 5-fluorouracil. Clin. Cancer Res. 8, 1107-1116 (2002).

79. Moliterni, A. et al. HER2 overexpression and doxorubicin in adjuvant chemotherapy for resectable breast cancer. J. Clin. Oncol. 21, 458-462 (2003).

80. Vera, R. et al. HER2 overexpression as a predictor of survival in a trial comparing adjuvant FAC and CMF in breast cancer. Proc. Am. Soc. Clin. Oncol. 18, 71 (1999).

81. Knoop, A. S. et al. Retrospective analysis of topoisomerase lla amplifications and deletions as predictive markers in primary breast cancer patients randomly assigned to cyclophosphamide, methotrexate, and fluorouracil or cyclophosphamide, epirubicin, and fluorouracil: Danish Breast Cancer Cooperative Group. J. Clin. Oncol. 23, 7483-7490 (2005).

82. Colozza, M. et al. HER2 overexpression as a predictive marker in a randomized trial comparing adjuvant cyclophosphamide/methotrexate/5-fluorouracil with epirubicin in patients with stage I/II breast cancer: long-term results. Clin. Breast Cancer 6, 253-259 (2005).

83. Pritchard, K. I. et al. HER2 and responsiveness of breast cancer to adjuvant chemotherapy. N. Engl. J. Med. 354, 2103-2111 (2006).

84. Bartlett, J. M. et al. Type 1 receptor tyrosine kinase profiles identify patients with enhanced benefit from anthracyclines in the BR9601 adjuvant breast cancer chemotherapy trial. J. Clin. Oncol. 26, 5027-5035 (2008).

85. Bartlett, J. M. et al. Predictive markers of anthracycline benefit: a prospectively planned analysis of the UK National Epirubicin Adjuvant Trial (NEAT/BR9601). Lancet Oncol. 11, 266-274 (2010).

86. Cheang, M. C. et al. Responsiveness of intrinsic subtypes to adjuvant anthracycline substitution in the NCIC.CTG MA.5 randomized trial. Clin. Cancer Res. 18, 2402-2412 (2012).

87. Di Leo, A. et al. HER2 and TOP2A as predictive markers for anthracyclinecontaining chemotherapy regimens as adjuvant treatment of breast cancer: a meta-analysis of individual patient data. Lancet Oncol. 12, 1134-1142 (2011).

88. Jensen, M. B. et al. The Prosigna 50-gene profile and responsiveness to adjuvant anthracycline-based chemotherapy in high-risk breast cancer patients. NPJ Breast Cancer 6, 7 (2020).

89. Gennari, A. et al. HER2 status and efficacy of adjuvant anthracyclines in early breast cancer: a pooled analysis of randomized trials. J. Natl Cancer Inst. 100, 14-20 (2008).

90. Dhesy-Thind, B. et al. HER2/neu in systemic therapy for women with breast cancer: a systematic review. Breast Cancer Res Treat. 109, 209-229 (2008).

91. Muss, H. B. et al. c-erbB-2 expression and response to adjuvant therapy in women with node-positive early breast cancer. N. Engl. J. Med. 330, 1260-1266 (1994).

92. Thor, A. D. et al. erbB-2, p53, and efficacy of adjuvant therapy in lymph nodepositive breast cancer. J. Natl Cancer Inst. 90, 1346-1360 (1998).

93. Dressler, L. G. et al. Comparison of HER2 status by fluorescence in situ hybridization and immunohistochemistry to predict benefit from dose escalation of adjuvant doxorubicin-based therapy in node-positive breast cancer patients. J. Clin. Oncol. 23, 4287-4297 (2005).

94. Hayes, D. F. et al. HER2 and response to paclitaxel in node-positive breast cancer. N. Engl. J. Med. 357, 1496-1506 (2007).

95. Arnould, L. et al. Epirubicin dose-response effect in node-positive breast cancer patients is independent of HER2 overexpression: 10-year retrospective analysis of French Adjuvant Study Group 05 trial. Breast Cancer Res Treat. 76, abstr. 538 (2003).

96. Del Mastro, L. et al. HER2 expression and efficacy of dose-dense anthracyclinecontaining adjuvant chemotherapy in breast cancer patients. Br. J. Cancer 93, 7-14 (2005).

97. Petit, T. et al. Chemotherapy response of breast cancer depends on HER-2 status and anthracycline dose intensity in the neoadjuvant setting. Clin. Cancer Res. 7, 1577-1581 (2001).

98. Fasching, P. A. et al. Final results of the ASG1-3 study, a randomized phase III study comparing a standard dose chemotherapy with epirubicin/cyclophosphamide and 
paclitaxel with a dose dense regimen with epirubicin and paclitaxel. Cancer Res. https://doi.org/10.1158/1538-7445.SABCS18-P1-13-01 (2019).

99. Kostopoulos, I. et al. Evaluation of the prognostic value of HER-2 and VEGF in breast cancer patients participating in a randomized study with dose-dense sequential adjuvant chemotherapy. Breast Cancer Res. Treat. 96, 251-261 (2006).

100. Rodenhuis, S. et al. Efficacy of high-dose alkylating chemotherapy in HER2/neunegative breast cancer. Ann. Oncol. 17, 588-596 (2006).

101. Early Breast Cancer Trialists' Collaborative, G. Increasing the dose intensity of chemotherapy by more frequent administration or sequential scheduling: a patient-level meta-analysis of 37298 women with early breast cancer in 26 randomised trials. Lancet 393, 1440-1452 (2019).

102. Hurvitz, S. \& McCann, K. 1 Online Resource) (Elsevier, 2018).

103. Press, M. F. et al. HER-2/neu gene amplification characterized by fluorescence in situ hybridization: poor prognosis in node-negative breast carcinomas. J. Clin. Oncol. 15, 2894-2904 (1997).

104. Press, M. F. et al. Evaluation of HER-2/neu gene amplification and overexpression: comparison of frequently used assay methods in a molecularly characterized cohort of breast cancer specimens. J. Clin. Oncol. 20, 3095-3105 (2002).

105. Benz, C. C. et al. Estrogen-dependent, tamoxifen-resistant tumorigenic growth of MCF-7 cells transfected with HER2/neu. Breast Cancer Res. Treat. 24, 85-95 (1992).

106. Pegram, M. D. et al. The effect of HER-2/neu overexpression on chemotherapeutic drug sensitivity in human breast and ovarian cancer cells. Oncogene 15, 537-547 (1997).

107. Orr, M. S., O'Connor, P. M. \& Kohn, K. W. Effects of c-erbB2 overexpression on the drug sensitivities of normal human mammary epithelial cells. J. Natl. Cancer Inst. 92, 987-994 (2000).

108. Konecny, G. et al. HER-2/neu overexpression and in vitro chemosensitivity to CMF and FEC in primary breast cancer. Breast Cancer Res. Treat. 69, 53-63 (2001).

109. Blum, J. L. et al. Anthracyclines in early breast cancer: the ABC trials-USOR 06090, NSABP B-46-I/USOR 07132, and NSABP B-49 (NRG Oncology). J Clin Oncol. https://doi.org/10.1200/JCO.2016.71.4147 (2017)

110. Nitz, U. et al. West German Study PlanB trial: adjuvant four cycles of epirubicin and cyclophosphamide plus docetaxel versus six cycles of docetaxel and cyclophosphamide in HER2-negative early breast cancer. J. Clin. Oncol. 37, 799-808 (2019).

111. Janni, W. et al. Pooled analysis of two randomized phase III trials (PlanB/SuccessC) comparing six cycles of docetaxel and cyclophosphamide to sequential anthracycline taxane chemotherapy in patients with intermediate and high risk HER2negative early breast cancer $(n=5,923)$. J. Clin. Oncol. 36, 522 (2018).

112. Mavroudis, D. et al. Dose-dense FEC followed by docetaxel versus docetaxel plus cyclophosphamide as adjuvant chemotherapy in women with HER2-negative, axillary lymph node-positive early breast cancer: a multicenter randomized study by the Hellenic Oncology Research Group (HORG). Ann. Oncol. 27, 1873-1878 (2016)

113. Kim, H. A. et al. Evaluation of the survival benefit of different chemotherapy regimens in patients with T1-2N0 triple-negative breast cancer. J. Breast Cancer 18, 271-278 (2015).

114. Sharma, P. et al. Efficacy of neoadjuvant carboplatin plus docetaxel in triplenegative breast cancer: combined analysis of two cohorts. Clin. Cancer Res. 23, 649-657 (2017)

115. Sharma, P. et al. Pathological response and survival in triple-negative breast cancer following neoadjuvant carboplatin plus docetaxel. Clin. Cancer Res. 24, 5820-5829 (2018).

116. Enriquez, D. et al. Improving pathological response in locally advanced triple negative breast cancer: Comparison between $\mathrm{CbD}$ and $\mathrm{AC}-\mathrm{T}$ regimens. J. Clin. Oncol. 35, abstr. 585 (2017).

117. Sharma, P. et al. Randomized phase II trial of anthracycline-free and anthracyclinecontaining neoadjuvant carboplatin chemotherapy regimens in stage I-III triplenegative breast cancer (NeoSTOP). Clin. Cancer Res. 27, 975-982 (2021).

118. Zhang, P. et al. Better pathologic complete response and relapse-free survival after carboplatin plus paclitaxel compared with epirubicin plus paclitaxel as neoadjuvant chemotherapy for locally advanced triple-negative breast cancer: a randomized phase 2 trial. Oncotarget 7, 60647-60656 (2016).

119. Najafi, S. et al. Phase II study of adjuvant docetaxel and carboplatin with/without doxorubicin and cyclophosphamide in triple negative breast cancer: a randomised controlled clinical trial. Contemp. Oncol. 21, 83-89 (2017).

120. Wang, J. et al. A randomized phase III trial comparing dose-dense epirubicin and cyclophosphamide (ECdd) followed by paclitaxel $(\mathrm{T})$ with paclitaxel plus carboplatin (PCdd) as adjuvant chemotherapy for early triple-negative breast cancer patients with high-recurrence risk. J. Clin. Oncol. 37, 528 (2019).

121. Du, F. et al. Carboplatin plus taxanes are non-inferior to epirubicin plus cyclophosphamide followed by taxanes as adjuvant chemotherapy for early triplenegative breast cancer. Breast Cancer Res. Treat. 182, 67-77 (2020).
122. Zhang, L. et al. Neoadjuvant docetaxel + carboplatin versus epirubicin+cyclophosphamide followed by docetaxel in triple-negative, early-stage breast cancer (NeoCART): results from a multicenter, randomized controlled, open-label, phase II trial. J. Clin. Oncol. 38, 586 (2020).

123. Withoff, S., De Jong, S., De Vries, E. G. \& Mulder, N. H. Human DNA topoisomerase II: biochemistry and role in chemotherapy resistance (review). Anticancer Res. 16, 1867-1880 (1996).

124. Roca, J. \& Wang, J. C. DNA transport by a type II DNA topoisomerase: evidence in favor of a two-gate mechanism. Cell 77, 609-616 (1994).

125. Järvinen, T. A. et al. Amplification and deletion of topoisomerase llalpha associate with ErbB-2 amplification and affect sensitivity to topoisomerase II inhibitor doxorubicin in breast cancer. Am. J. Pathol. 156, 839-847 (2000).

126. Smith, K., Houlbrook, S., Greenall, M., Carmichael, J. \& Harris, A. L. Topoisomerase II alpha co-amplification with erbB2 in human primary breast cancer and breast cancer cell lines: relationship to m-AMSA and mitoxantrone sensitivity. Oncogene 8, 933-938 (1993).

127. Withoff, S. et al. Selection of a subpopulation with fewer DNA topoisomerase II alpha gene copies in a doxorubicin-resistant cell line panel. Br. J. Cancer 74, 502-507 (1996).

128. Jarvinen, T. A., Tanner, M., Barlund, M., Borg, A. \& Isola, J. Characterization of topoisomerase II alpha gene amplification and deletion in breast cancer. Genes Chromosomes Cancer 26, 142-150 (1999).

129. Zaczek, A. et al. Prognostic value of TOP2A gene amplification and chromosome 17 polysomy in early breast cancer. Pathol. Oncol. Res. 18, 885-894 (2012).

130. Zaczek, A. J. et al. Prognostic significance of TOP2A gene dosage in HER-2negative breast cancer. Oncologist 17, 1246-1255 (2012).

131. Coon, J. S. et al. Amplification and overexpression of topoisomerase llalpha predict response to anthracycline-based therapy in locally advanced breast cancer. Clin. Cancer Res. 8, 1061-1067 (2002).

132. Durbecq, V. et al. Comparison of topoisomerase-llalpha gene status between primary breast cancer and corresponding distant metastatic sites. Breast Cancer Res. Treat. 77, 199-204 (2003).

133. Cardoso, F. et al. Correlation between complete response to anthracyclinebased chemotherapy and topoisomerase II-alpha gene amplification and protein overexpression in locally advanced/metastatic breast cancer. Int. J. Oncol. 24, 201-209 (2004).

134. Bouchalova, K. et al. Analysis of ERBB2 and TOP2A gene status using fluorescence in situ hybridization versus immunohistochemistry in localized breast cancer. Neoplasma 53, 393-401 (2006).

135. Hicks, D. G. et al. The incidence of topoisomerase II-alpha genomic alterations in adenocarcinoma of the breast and their relationship to human epidermal growth factor receptor-2 gene amplification: a fluorescence in situ hybridization study. Hum. Pathol. 36, 348-356 (2005).

136. Desmedt, C. et al. Multifactorial approach to predicting resistance to anthracyclines. J. Clin. Oncol. 29, 1578-1586 (2011).

137. Press, M. F. et al. Alteration of topoisomerase II-alpha gene in human breast cancer: association with responsiveness to anthracycline-based chemotherapy. J. Clin. Oncol. 29, 859-867 (2011).

138. Callagy, G. et al. Identification and validation of prognostic markers in breast cancer with the complementary use of array-CGH and tissue microarrays. $J$. Pathol. 205, 388-396 (2005).

139. Arriola, E. et al. Topoisomerase II alpha amplification may predict benefit from adjuvant anthracyclines in HER2 positive early breast cancer. Breast Cancer Res. Treat. 106, 181-189 (2007).

140. Fasching, P. A. et al. HER2 and TOP2A amplification in a hospital-based cohort of breast cancer patients: associations with patient and tumor characteristics. Breast Cancer Res. Treat. 145, 193-203 (2014).

141. Olsen, K. E. et al. Amplification of HER2 and TOP2A and deletion of TOP2A genes in breast cancer investigated by new FISH probes. Acta Oncol. 43, 35-42 (2004).

142. Chen, J. R. et al. Amplification of HER2 and TOP2A and deletion of TOP2A genes in a series of Taiwanese breast cancer. Medicine 96, e5582 (2017).

143. Tubbs, R. et al. Outcome of patients with early-stage breast cancer treated with doxorubicin-based adjuvant chemotherapy as a function of HER2 and TOP2A status. J. Clin. Oncol. 27, 3881-3886 (2009).

144. Harris, L. N. et al. Topoisomerase II\{alpha\} amplification does not predict benefit from dose-intense cyclophosphamide, doxorubicin, and fluorouracil therapy in HER2-amplified early breast cancer: results of CALGB 8541/150013. J. Clin. Oncol. 27, 3430-3436 (2009).

145. Mueller, R. E., Parkes, R. K., Andrulis, I. \& O'Malley, F. P. Amplification of the TOP2A gene does not predict high levels of topoisomerase II alpha protein in human breast tumor samples. Genes Chromosomes Cancer 39, 288-297 (2004).

146. Bofin, A. M., Ytterhus, B. \& Hagmar, B. M. TOP2A and HER-2 gene amplification in fine needle aspirates from breast carcinomas. Cytopathology 14, 314-319 (2003). 
147. Nielsen, K. V. et al. The value of TOP2A gene copy number variation as a biomarker in breast cancer: update of DBCG trial 89D. Acta Oncol. 47, 725-734 (2008).

148. Fountzilas, G. et al. Evaluation of the prognostic role of centromere 17 gain and HER2/topoisomerase II alpha gene status and protein expression in patients with breast cancer treated with anthracycline-containing adjuvant chemotherapy: pooled analysis of two Hellenic Cooperative Oncology Group (HeCOG) phase III trials. BMC Cancer 13, 163 (2013).

149. Brase, J. C. et al. ERBB2 and TOP2A in breast cancer: a comprehensive analysis of gene amplification, RNA levels, and protein expression and their influence on prognosis and prediction. Clin. Cancer Res. 16, 2391-2401 (2010).

150. O'Malley, F. P. et al. Topoisomerase II alpha and responsiveness of breast cancer to adjuvant chemotherapy. J. Natl Cancer Inst. 101, 644-650 (2009).

151. Engstrom, M. J., Ytterhus, B., Vatten, L. J., Opdahl, S. \& Bofin, A. M. TOP2A gene copy number change in breast cancer. J. Clin. Pathol. 67, 420-425 (2014).

152. Konecny, G. E. et al. Association between HER2, TOP2A, and response to anthracycline-based preoperative chemotherapy in high-risk primary breast cancer. Breast Cancer Res. Treat. 120, 481-489 (2010).

153. Schindlbeck, C. et al. Topoisomerase llalpha expression rather than gene amplification predicts responsiveness of adjuvant anthracycline-based chemotherapy in women with primary breast cancer. J. Cancer Res. Clin. Oncol. 136, 1029-1037 (2010).

154. Miettinen, H. E. et al. High topoisomerase Ilalpha expression associates with high proliferation rate and and poor prognosis in oligodendrogliomas. Neuropathol. Appl. Neurobiol. 26, 504-512 (2000).

155. Nakopoulou, L. et al. DNA topoisomerase Il-alpha immunoreactivity as a marker of tumor aggressiveness in invasive breast cancer. Pathobiology 68, 137-143 (2000).

156. Willman, J. H. \& Holden, J. A. Immunohistochemical staining for DNA topoisomerase II-alpha in benign, premalignant, and malignant lesions of the prostate. Prostate 42, 280-286 (2000)

157. Brustmann, H. \& Naude, S. Expression of topoisomerase Ilalpha, Ki-67, proliferating cell nuclear antigen, p53, and argyrophilic nucleolar organizer regions in vulvar squamous lesions. Gynecol. Oncol. 86, 192-199 (2002).

158. Romero, A. et al. Assessment of topoisomerase II alpha status in breast cancer by quantitative PCR, gene expression microarrays, immunohistochemistry, and fluorescence in situ hybridization. Am. J. Pathol. 178, 1453-1460 (2011).

159. Nikolenyi, A. et al. Tumor topoisomerase II alpha status and response to anthracycline-based neoadjuvant chemotherapy in breast cancer. Oncology 80, 269-277 (2011).

160. Nikolenyi, A. et al. Tumour topoisomerase II alpha protein expression and outcome after adjuvant dose-dense anthracycline-based chemotherapy. Pathol. Oncol. Res. 18, 61-68 (2012).

161. Isaacs, R. J. et al. Physiological regulation of eukaryotic topoisomerase II. Biochim. Biophys. Acta 1400, 121-137 (1998).

162. Isaacs, R. J., Harris, A. L. \& Hickson, I. D. Regulation of the human topoisomerase llalpha gene promoter in confluence-arrested cells. J. Biol. Chem. 271, 16741-16747 (1996).

163. Qiao, J. H., Jiao, D. C., Lu, Z. D., Yang, S. \& Liu, Z. Z. Clinical significance of topoisomerase $2 \mathrm{~A}$ expression and gene change in operable invasive breast cancer. Tumour Biol. 36, 6833-6838 (2015).

164. Erber, R. et al. Predictive role of HER2/neu, topoisomerase-II-alpha, and tissue inhibitor of metalloproteinases (TIMP-1) for response to adjuvant taxane-based chemotherapy in patients with intermediate-risk breast cancer: results from the WSG-AGO EC-Doc trial. Breast Cancer Res. Treat. 150, 279-288 (2015).

165. Durbecq, V. et al. Topoisomerase-II alpha expression as a predictive marker in a population of advanced breast cancer patients randomly treated either with single-agent doxorubicin or single-agent docetaxel. Mol. Cancer Ther. 3, 1207-1214 (2004).

166. Di Leo, A. et al. p-53 gene mutations as a predictive marker in a population of advanced breast cancer patients randomly treated with doxorubicin or docetaxel in the context of a phase III clinical trial. Ann. Oncol. 18, 997-1003 (2007).

167. Chen, S. et al. The predictive and prognostic significance of pre- and posttreatment topoisomerase Ilalpha in anthracycline-based neoadjuvant chemotherapy for local advanced breast cancer. Eur. J. Surg. Oncol. 39, 619-626 (2013)

168. O'Malley, F. P. et al. Topoisomerase II alpha protein and responsiveness of breast cancer to adjuvant chemotherapy with CEF compared to CMF in the NCIC CTG randomized MA.5 adjuvant trial. Breast Cancer Res. Treat. 128, 401-409 (2011).

169. Mukherjee, A. et al. Topo2alpha protein expression predicts response to anthracycline combination neo-adjuvant chemotherapy in locally advanced primary breast cancer. Br. J. Cancer 103, 1794-1800 (2010).

170. Li, X. R. et al. ER, PgR, HER-2, Ki-67, topoisomerase llalpha, and nm23-H1 proteins expression as predictors of pathological complete response to neoadjuvant chemotherapy for locally advanced breast cancer. Med. Oncol. 28(Suppl. 1), S48-S54 (2011).

171. Rody, A. et al. Gene expression profiling of breast cancer patients treated with docetaxel, doxorubicin, and cyclophosphamide within the GEPARTRIO trial: HER2 , but not topoisomerase II alpha and microtubule-associated protein tau, is highly predictive of tumor response. Breast 16, 86-93 (2007).

172. Orlando, L. et al. Topoisomerase Ilalpha gene status and prediction of pathological complete remission after anthracycline-based neoadjuvant chemotherapy in endocrine non-responsive Her2/neu-positive breast cancer. Breast 17, 506-511 (2008).

173. Wang, J. et al. TOP2A amplification in breast cancer is a predictive marker of anthracycline-based neoadjuvant chemotherapy efficacy. Breast Cancer Res. Treat. 135, 531-537 (2012)

174. Almeida, D. et al. Topoisomerase II-alfa gene as a predictive marker of response to anthracyclines in breast cancer. Pathol. Res. Pr. 210, 675-679 (2014).

175. Tanner, M. et al. Topoisomerase llalpha gene amplification predicts favorable treatment response to tailored and dose-escalated anthracycline-based adjuvant chemotherapy in HER-2/neu-amplified breast cancer: Scandinavian Breast Group Trial 9401. J. Clin. Oncol. 24, 2428-2436 (2006).

176. Bartlett, J. M. et al. Predicting anthracycline benefit: TOP2A and CEP17-not only but also. J. Clin. Oncol. 33, 1680-1687 (2015).

177. Corzo, C. et al. Does polysomy of chromosome 17 have a role in ERBB2 and topoisomerase llalpha expression? Gene, mRNA and protein expression: a comprehensive analysis. Tumour Biol. 28, 221-228 (2007).

178. Pritchard, K. I. et al. Chromosome 17 centromere (CEP17) duplication as a predictor of anthracycline response: evidence from the NCIC Clinical Trials Group (NCIC CTG) MA.5 trial. Breast Cancer Res. Treat. 131, 541-551 (2012).

179. Nielsen, K. V. et al. Lack of independent prognostic and predictive value of centromere 17 copy number changes in breast cancer patients with known HER2 and TOP2A status. Mol. Oncol. 6, 88-97 (2012).

180. Stephens, P. J. et al. Massive genomic rearrangement acquired in a single catastrophic event during cancer development. Cell 144, 27-40 (2011).

181. Vasmatzis, G. et al. Chromoanasynthesis is a common mechanism that leads to ERBB2 amplifications in a cohort of early stage HER2(+) breast cancer samples. BMC Cancer 18, 738 (2018).

182. Cortes-Ciriano, I. et al. Comprehensive analysis of chromothripsis in 2,658 human cancers using whole-genome sequencing. Nat. Genet. 52, 331-341 (2020).

183. Shoshani, O. et al. Chromothripsis drives the evolution of gene amplification in cancer. Nature 591, 137-141 (2021).

184. Ejlertsen, B. et al. Adjuvant cyclophosphamide and docetaxel with or without epirubicin for early TOP2A-normal breast cancer: DBCG 07-READ, an open-label, phase III, randomized trial. J. Clin. Oncol. 35, 2639-2646 (2017).

185. Slamon, D. J. et al. Use of chemotherapy plus a monoclonal antibody against HER2 for metastatic breast cancer that overexpresses HER2. N. Engl. J. Med. 344, 783-792 (2001).

186. Joensuu, $\mathrm{H}$. et al. Adjuvant docetaxel or vinorelbine with or without trastuzumab for breast cancer. N. Engl. J. Med. 354, 809-820 (2006).

187. Piccart-Gebhart, M. J. et al. Trastuzumab after adjuvant chemotherapy in HER2positive breast cancer. N. Engl. J. Med. 353, 1659-1672 (2005).

188. Romond, E. H. et al. Trastuzumab plus adjuvant chemotherapy for operable HER2-positive breast cancer. N. Engl. J. Med. 353, 1673-1684 (2005).

189. Slamon, D. et al. Adjuvant trastuzumab in HER2-positive breast cancer. N. Engl. J. Med. 365, 1273-1283 (2011).

190. Pegram, M. D. \& Slamon, D. J. Combination therapy with trastuzumab (Herceptin) and cisplatin for chemoresistant metastatic breast cancer: evidence for receptor-enhanced chemosensitivity. Semin. Oncol. 26, 89-95 (1999).

191. Pegram, M. et al. Inhibitory effects of combinations of HER-2/neu antibody and chemotherapeutic agents used for treatment of human breast cancers. Oncogene 18, 2241-2251 (1999).

192. Pegram, M. D. et al. Rational combinations of trastuzumab with chemotherapeutic drugs used in the treatment of breast cancer. J. Natl Cancer Inst. 96, 739-749 (2004).

193. Schneeweiss, A. et al. Long-term efficacy analysis of the randomised, phase II TRYPHAENA cardiac safety study: evaluating pertuzumab and trastuzumab plus standard neoadjuvant anthracycline-containing and anthracycline-free chemotherapy regimens in patients with HER2-positive early breast cancer. Eur. J. Cancer 89, 27-35 (2018).

194. van Ramshorst, M. S. et al. Neoadjuvant chemotherapy with or without anthracyclines in the presence of dual HER2 blockade for HER2-positive breast cancer (TRAIN-2): a multicentre, open-label, randomised, phase 3 trial. Lancet Oncol. 19, 1630-1640 (2018).

195. van der Voort, A. et al. Three-year follow-up of neoadjuvant chemotherapy with or without anthracyclines in the presence of dual HER2-blockade for HER2- 
positive breast cancer (TRAIN-2): a randomized phase III trial. J. Clin. Oncol. 38, 501 (2020).

196. Slamon, D. J. et al. Ten year follow-up of BCIRG-006 comparing doxorubicin plus cyclophosphamide followed by docetaxel $(A C \rightarrow T)$ with doxorubicin plus cyclophosphamide followed by docetaxel and trastuzumab $(A C \rightarrow T H)$ with docetaxel, carboplatin and trastuzumab (TCH) in HER2 + early breast cancer. Cancer Res. 76, abstr. S5-S04 (2015).

197. National Comprehensive Cancer Network Clinical Practice Guidelines in Oncology: Breast Cancer. v3.2021. https://www.nccn.org/professionals/physician_gls/ pdf/breast.pdf.

198. Romond, E. H. et al. Seven-year follow-up assessment of cardiac function in NSABP B-31, a randomized trial comparing doxorubicin and cyclophosphamide followed by paclitaxel (ACP) with ACP plus trastuzumab as adjuvant therapy for patients with node-positive, human epidermal growth factor receptor 2-positive breast cancer. J. Clin. Oncol. 30, 3792-3799 (2012).

199. Advani, P. P., Ballman, K. V., Dockter, T. J., Colon-Otero, G. \& Perez, E. A. Longterm cardiac safety analysis of NCCTG N9831 (Alliance) adjuvant trastuzumab trial. J. Clin. Oncol. 34, 581-587 (2016).

200. Felix, C. A., Lange, B. J., Hosler, M. R., Fertala, J. \& Bjornsti, M. A. Chromosome band 11q23 translocation breakpoints are DNA topoisomerase II cleavage sites. Cancer Res. 55, 4287-4292 (1995).

201. Broeker, P. L. et al. Distribution of $11 q 23$ breakpoints within the MLL breakpoint cluster region in de novo acute leukemia and in treatment-related acute myeloid leukemia: correlation with scaffold attachment regions and topoisomerase II consensus binding sites. Blood 87, 1912-1922 (1996).

202. Tallman, M. S. et al. Leukemogenic potential of adjuvant chemotherapy for early-stage breast cancer: the Eastern Cooperative Oncology Group experience. J. Clin. Oncol. 13, 1557-1563 (1995).

\section{ACKNOWLEDGEMENTS}

The authors would like to acknowledge background research assistance from Ryan Ponec, MD; support from the UCLA Jonsson Comprehensive Cancer Center (S.A.H., D.J.S., J.A.G. and N.P.M.), the Marni Levine Memorial Research Award (S.A.H.), USC Norris Comprehensive Cancer Center (M.F.P., NCl Cancer Center Support Grant P30 CA014089), the Breast Cancer Research Foundation (M.F.P., BCRF-18-132), Tower Cancer Research Foundation (M.F.P., Jessica M. Berman Senior Investigator Award), and a gift from Dr. Richard Balch (M.F.P.).

\section{AUTHOR CONTRIBUTIONS}

S.A.H. and D.J.S. conceived and designed the work, S.A.H. drafted the original manuscript, N.P.M. added to the manuscript and drafted the tables, S.A.H., D.J.S., N.P. M., A.B., M.F.P., M.P., J.P.C., P.A.F., B.E., E.H.Y., J.A.G. and D.J.S. provided substantial contribution to the design of the work as well as revisions and additions to the manuscript, provided final approval, and are accountable for all aspects of the work.

\section{COMPETING INTERESTS}

S.A.H.: Contracted research paid to institution: Ambrx, Amgen, AstraZeneca, Arvinas, Bayer, Cytomx, Daiichi Sankyo, Dignitana, Genentech/Roche, Gilead, GSK, Immunomedics, Lilly, Macrogenics, Novartis, Pfizer, OBI Pharma, Pieris, PUMA, Radius, Samumed, Sanofi, Seattle Genetics/Seagen, Zymeworks, Phoenix Molecular Designs, Ltd. Travel expenses: Lilly. Uncompensated consulting: 4DPharma, Ambrx, Amgen, Artios, Arvinas, Daiichi Sankyo, Dantari, Genentech/Roche, Immunomedics, Macrogenics, Lilly, Novartis, Pieris, Pyxis, Seagen, N.P.M.: Research funding from Novartis, Daiichi Sankyo, and Dizal, advisory board honorarium from Novartis, Daiichi Sankyo, Biotheranostics, and Genomic Health, consulting honorarium from Novartis and Daiichi Sankyo, travel accommodation from TRIO,
Daiichi Sankyo, and Roche, as well as speaking honorarium from Novartis. A.B.: Consulting: Immunomedics, Pfizer, Novartis, Genentech/Roche, Merck, Radius Health, Spectrum Pharma, Taiho Pharma, Biothernostics Inc., Sanofi, Daich Pharma, Puma; personal fees from Biothernostics Inc., Pfizer, Novartis, Genentech/ Roche, Merck, Radius Health, Immunomedics, Spectrum Pharma, Taiho Pharma, Sanofi, Daiichi Pharma, Puma; grants paid to the institution from Genentech/ Roche, Novartis, Pfizer, Merck, Sanofi, Radius Health, Immunomedics, Mersana, Innocrin, Biothernostics Inc. M.F.P.: Contracted research: Cepheid; Eli Lilly \& Company; Novartis Pharmaceuticals; F. Hoffmann-La Roche Ltd; Puma; Consulting or advisory role with honoraria: AstraZeneca; Biocartis SA; Cepheid, Eli Lilly \& Company, USA, LLC; Merck \& Co; Puma Biotechnology; Zymeworks Inc. Expert testimony: Amgen, Inc. Private equity: TORL Biotherapeutics, LLC. M.P.: Honoraria Roche/Genentech; Sponsored Research: Stanford Cancer Institute, Parker Institute for Cancer Immunotherapy, Pfizer, G1 Therapeutics, Zymeworks, Daiichi Sankyo, Odonate, Bolt Biotheranostics. J.P.C.: Research funding (to institution): Eisai, Puma Biotechnology, Roche, Boehringer Ingelheim; Employment: OncoMark, Ltd.; Honoraria: Eisai, Puma Biotechnology; MSD Oncology, Pfizer, G1 Therapeutics; Novartis; Speaker's Bureau: Boehringer Ingelheim, Genomic Health, Roche, Pfizer Shares: OncoMark Ltd; Travel and accommodation expenses: Pfizer, MSD, Abbvie, Astrazeneca, Novartis. P.A.F.: Research funding to institution: Biontech, Cepheid. Consulting fees: Novartis, Pfizer, Daiichi Sankyo, AstraZeneca, Eisai, Merck Sharp \& Dohme, Lilly, Pierre Fabre, SeaGen, Roche, Hexal, Agendia. B.E.: Research funding to institution: AstraZeneca, MSD, NanoString Technologies, Novartis, Oncology Venture, Pfizer, Roche, and Samsung. E.H.Y.: research funding from CSL Behring. J. A.G.: no conflicts to report. D.J.S.: Advisory board membership: Biomarin; Consultancy: Lilly, Novartis, Pfizer, Seagen; Equity ownership: Amgen, Biomarin, Merck, Sharp \& Dohme, Pfizer, Seagen, Vertex; Honorarium: Novartis; Research funding paid to institution: Novartis, Pfizer, Seagen; Speaker's Bureau: Novartis; Travel expenses: Biomarin, Novartis, Pfizer.

\section{ADDITIONAL INFORMATION}

Supplementary information The online version contains supplementary material available at https://doi.org/10.1038/s41523-021-00342-5.

Correspondence and requests for materials should be addressed to Sara Alsterlind Hurvitz.

Reprints and permission information is available at http://www.nature.com/ reprints

Publisher's note Springer Nature remains neutral with regard to jurisdictional claims in published maps and institutional affiliations.

Open Access This article is licensed under a Creative Commons Attribution 4.0 International License, which permits use, sharing, adaptation, distribution and reproduction in any medium or format, as long as you give appropriate credit to the original author(s) and the source, provide a link to the Creative Commons license, and indicate if changes were made. The images or other third party material in this article are included in the article's Creative Commons license, unless indicated otherwise in a credit line to the material. If material is not included in the article's Creative Commons license and your intended use is not permitted by statutory regulation or exceeds the permitted use, you will need to obtain permission directly from the copyright holder. To view a copy of this license, visit http://creativecommons. org/licenses/by/4.0/.

(c) The Author(s) 2021 